\title{
Multiphase-field modeling of spinodal decomposition during intercalation in an Allen-Cahn framework
}

\author{
Simon Daubner $\odot,{ }^{1,{ }^{*}}$ P G Kubendran Amos $\odot,{ }^{1,2}$ Ephraim Schoof $\odot,{ }^{1}$ Jay Santoki, ${ }^{1}$ Daniel Schneider,${ }^{1,3}$ and Britta Nestler ${ }^{1,3}$ \\ ${ }^{1}$ Institute of Applied Materials-Computational Materials Science, Karlsruhe Institute of Technology, \\ Strasse am Forum 7, 76131 Karlsruhe, Germany \\ ${ }^{2}$ Department of Metallurgical and Materials Engineering, National Institute of Technology, Tiruchirappalli 620015, Tamil Nadu, India \\ ${ }^{3}$ Institute of Digital Materials Science, Karlsruhe University of Applied Sciences, Moltkestr. 30, 76133 Karlsruhe, Germany
}

(Received 30 September 2020; accepted 3 March 2021; published 18 March 2021)

\begin{abstract}
Owing to its unique thermodynamical description, phase separation has largely been modeled in the Cahn-Hilliard framework. In the present work, as a computationally efficient alternative, a multicomponent, multiphase-field model operating in Allen-Cahn framework is presented and subsequently employed to simulate spinodal decomposition. Stability analysis shows that the formulation can cover the effect of phase separation while simplifying the extension to multiple phases and incorporation of additional driving forces. Computational efficiency of the proposed approach is compared with the conventional technique by modeling intercalation in a representative one-dimensional domain. Moreover, intercalation within a multigrain system involving multiparticle interaction is studied. Our results suggest initiation of phase transformation at higher order junctions as well as a grain-by-grain intercalation behavior in a two-phase cathode material such as the well-studied $\mathrm{LiFePO}_{4}$.
\end{abstract}

DOI: 10.1103/PhysRevMaterials.5.035406

\section{INTRODUCTION}

Microstructural evolutions include regular first-order phase transformations such as solidification as well as second-order continuous phase separations. For instance, some state-of-theart cathode materials in lithium ion battery systems exhibit phase separation during charging which influences their performance significantly. Therefore, attempts have been made to investigate the second-order phase transition in a multiphysics framework which encompasses electrical, chemical, and mechanical driving forces.

Originally introduced to the field of battery simulation by the studies of Han et al. [1] and Guyer et al. [2], the phase-field method is well established nowadays and has been applied in numerous works investigating electrodeposition [3-5] and intercalation in phase separating electrode materials [6-23]. Technically relevant cathode materials such as $\mathrm{LiFePO}_{4}$ (LFP) and the related $\mathrm{NaFePO}_{4}$ exhibit phase separation during charge and discharge which has been shown experimentally [24-26] and furthermore predicted by first-principles calculations $[27,28]$. The modeling thereof has been realized by the core-shell model [29] which prescribes an interface according to the Stefan problem and alternatively, by the phase-field method, which accounts for phase separation in a more natural manner [1]. The first work was limited to pure diffusion in one dimension [1], but applicability of the method was proven. Singh et al. [6] predicted intercalation waves by accounting for diffusion anisotropy and a surface reaction rate given by the Butler-Volmer (BV) equation.

Different focuses have been set since, one being the AllenCahn reaction model [30], which is based on the assumption of surface reaction limitation and fast bulk diffusion in

\footnotetext{
*simon.daubner@kit.edu
}

one-dimensional (1D) channels for LFP. This approach has been used to study the suppression of phase separation for high fluxes [7] and the pattern formation on an active facet of nanosized single crystals including coherency strain [8]. In a recent work [9], pattern formation depending on particle size was studied. Another approach, often referred to as the Cahn-Hilliard reaction model [30], uses the BV equation as a boundary condition while computing the flux inside the particle based on the conservative Cahn-Hilliard equation. Tang et al. [10] showed that the interface evolution is dependent on both surface flux and bulk diffusion. The recent works of Hong et al. [11,12] show how larger overpotentials shift the interface motion from surface reaction limitation to a hybrid or mainly bulk-diffusion limited case.

Huttin and Kamlah [13] studied intercalation in spherical particles reduced to one dimension by radial symmetry with constant boundary flux. Their original work was based on small deformations and later extended to large deformation mechanics [14]. Subsequent studies comprise the intercalation simulation in $\mathrm{NaFePO}_{4}$, a promising candidate for post-lithium batteries [15], and a comparison of miscibility gaps for various cathode materials [16]. Zhao et al. [17] introduced concentration-dependent elastic properties and performed simulations in spheroidal three-dimensional particles. In their later work [18], surface reaction was accounted for by the BV equation rather than constant flux, and diffusion-induced cracking was investigated. All these works focus on the influence of strongly coupled chemomechanics on diffusion and are characterized by sophisticated treatment of large deformation mechanics with the finite element method.

Other studies attempted to account for more realistic particle shapes. For example, Santoki et al. [19] studied the intercalation in an arbitrarily shaped particle in three dimensions. Constant boundary flux has been applied based on the 
smoothed boundary method, which has also been previously used to study spherical particles in two dimensions [20]. More complex geometries have been studied, such as the agglomerate of three spherical particles neglecting internal interfaces [21] and two touching nanowires [22]. Wu et al. [23] performed simulations with particle shapes reconstructed from x-ray tomography. They discuss the importance of realistic shapes as higher stress arises during insertion compared to simulations with idealized particles.

Since experimental observations provide evidence that multiparticle interaction noticeably affects the intercalation behavior [31], the need for extending existing techniques to multiparticle systems has been reported [32]. So far, phase-field simulations of intercalation are restricted to single crystals or single particles. Possible reasons are, first of all, the limitations linked to the Cahn-Hilliard approach used in basically all phase-field intercalation simulations including spinodal decomposition. The underlaying evolution equation is a fourth-order partial differential equation (PDE) which is computationally challenging. Furthermore, if the numerical interface width is set to reproduce the physical interface in the range of nanometers, this smallest resolved scale limits simulations to single nanoparticles with diameters in the range of $20-500 \mathrm{~nm}$. Secondly, the models reviewed above are formulated in a single-phase framework. Fleck et al. [33] and Santoki et al. [19] introduced an order parameter differentiating between particle and electrolyte for spatial parametrization but neglecting the ion transport due to electrochemical reaction between the two. Furthermore, both studies are limited to a single particle. From the discussion above, we identify two important steps paving the way to more realistic simulations. The modeling approach should be multiphase in the sense that it is capable of describing multiple particles with varying material properties surrounded by electrolytes. Secondly, a thermodynamically consistent formulation of the electrochemical reaction occurring at the interface between an active material and electrolyte is needed in terms of the phase-field method. The first is within the scope of this paper.

We therefore propose the use of an Allen-Cahn multiphasefield formulation coupled with conservative concentration evolution. Starting from a free-energy functional for multiphase systems [34], we introduce energetically decoupled interfacial and bulk energy contributions to avoid interfacial excess energy. This approach allows the width of the diffuse interface to be rescaled for numerical efficiency in the case of larger computational domains. The interfacial free-energy formulation from Nestler et al. [35] is used due to its ability to scale interfacial energy and width independently. The chemical free-energy density and consistent treatment of concentration evolution is based on the works of Plapp [36] as well as Choudhury and Nestler [37]. These works are based on a grand-potential formulation, but evolution equations can also be derived starting from a free-energy functional [38]. This approach is compatible with elastic driving forces based on jump conditions $[39,40]$ and has successfully been used for computation of first -order phase transformations [41]. The ability of this model formulation to account for spinodal phase separating behavior has recently been shown by Aagesen et al. [42], though it had previously been considered impossible due to the requirement of convex free-energy functions [36]. The aim of this study is to demonstrate the potentiality of modeling phase separation within the proposed framework and compare it with the model by Cahn and Hilliard [43] with regard to two-phase intercalation processes in $\mathrm{LiFePO}_{4}$. Parallelization using Message Passing Interface for computational efficiency allows for simulation of large-scale systems [44].

This paper is organized as follows. Section II presents two modeling approaches, both of which are started from a freeenergy functional, followed by a discussion of the free-energy density contributions and the evolution equations of primary variables of the system. The fitting of thermodynamic data by appropriate choice of the respective model parameters is performed in Sec. III. In this section, we also examine the stability of both model formulations with regard to the spontaneous separation of phases dependent on composition. The theoretical predictions are then studied further with an example system in Sec. IV A, followed by application to modeling intercalation in a multiparticle system in Sec. IV B. In Sec. V, the conclusion and future applications are discussed.

\section{MODEL FORMULATION IN CAHN-HILLIARD AND ALLEN-CAHN FRAMEWORKS}

\section{A. Cahn-Hilliard approach}

The model presented in this section stems from the seminal work of Cahn and Hilliard [43] but is generalized to $K$ chemical species with molar fractions $\boldsymbol{c}=\left\{c_{1}, \ldots, c_{i}, \ldots, c_{K}\right\}^{T}$. The total free energy for a nonuniform system can, in first approximation, be expressed as

$$
\mathcal{F}^{\mathrm{CH}}(\boldsymbol{c}, \nabla \boldsymbol{c})=\int_{V} f_{\text {grad }}^{\mathrm{CH}}(\nabla \boldsymbol{c})+f_{\text {chem }}^{\mathrm{CH}}(\boldsymbol{c}) d V
$$

consisting of two contributions, namely, the free chemical energy and a gradient energy [43]. In a multicomponent framework, several choices of free-energy contributions are possible but consistency with the binary case has to be ensured [45]. The gradient energy with coefficient $\kappa$ takes the form

$$
f_{\text {grad }}^{\mathrm{CH}}(\nabla \boldsymbol{c})=\kappa \sum_{i}^{K}\left|\nabla c_{i}\right|^{2},
$$

and following Refs. $[13,17,19]$, the regular solution theory is used to express the chemical free energy of the system

$$
f_{\text {chem }, \mathrm{RS}}^{\mathrm{CH}}(\boldsymbol{c})=\frac{R}{V_{\mathrm{m}}} T \sum_{i}^{K} c_{i} \ln \left(c_{i}\right)+\sum_{i}^{K} \sum_{j}^{K} c_{i} \chi_{i j} c_{j},
$$

where $R$ denotes the gas constant, $V_{\mathrm{m}}$ the molar volume, $T$ the temperature, and $\chi_{i j}$ the matrix of interaction parameters. In the following, all considerations refer to this specific choice of free energy though the transfer of the analysis performed in Secs. III A and III B to other formulations is straightforward. The evolution of composition can be computed by a set of $K-1$ equations of Cahn-Hilliard type,

$$
\frac{\partial c_{i}}{\partial t}=\nabla \cdot\left[L_{i j}(\boldsymbol{c}) \nabla \mu_{j}(T, \boldsymbol{c}, \boldsymbol{\phi})\right]
$$

with the mobility matrix $L_{i j}(\boldsymbol{c})=\frac{V_{\mathrm{m}}}{R} D_{i} c_{i}\left(\delta_{i j}-c_{j}\right)$, diffusion coefficients $D_{i}$ and $(\nabla \cdot)$ denoting the divergence operator. The last mole fraction can be determined by the sum constraint 
$c_{K}=1-\sum_{i}^{K-1} c_{i}$. The chemical potential $\mu_{i}$ of species $i$ can be expressed as the sum $\mu_{i}=\mu_{i}^{\text {grad }}+\mu_{i}^{\text {chem }}$ of the two contributions

$$
\begin{gathered}
\mu_{i}^{\mathrm{grad}}=-\nabla \cdot \frac{\partial f_{\mathrm{grad}}^{\mathrm{CH}}(\nabla \boldsymbol{c})}{\partial \nabla c_{i}}=-2 \kappa \nabla^{2} c_{i}, \\
\mu_{i}^{\mathrm{chem}}=\frac{\partial f_{\mathrm{chem}}^{\mathrm{CH}}(\boldsymbol{c})}{\partial c_{i}}=\frac{R}{V_{\mathrm{m}}} T\left[\ln \left(c_{i}\right)+1\right]+2 \sum_{j}^{K} \chi_{i j} c_{j} .
\end{gathered}
$$

The resulting evolution equation is fourth order in $c$ :

$$
\begin{aligned}
\frac{\partial c_{i}}{\partial t}= & \nabla \cdot\left[D_{i} c_{i} \sum_{j}^{K}\left(\delta_{i j}-c_{j}\right)\right. \\
& \left.\times \nabla\left\{T\left[\ln \left(c_{j}\right)+1\right]+2 \frac{V_{\mathrm{m}}}{R} \sum_{k}^{K} \chi_{j k} c_{k}-2 \kappa \nabla^{2} c_{j}\right\}\right] .
\end{aligned}
$$

\section{B. Allen-Cahn approach with concentration conservation}

The Allen-Cahn equation is another evolutionary relation often used in the context of microstructure simulations. In combination with the grand-chemical potential approach introduced by Plapp [36] and Choudhury and Nestler [37], conservation of mass for species $i$ combined with the nonconserved evolution of phases can be simulated. The decoupling of interfacial and bulk contributions eliminates the chemical excess to the interface energy which is particularly important in the context of numerically efficient computations of larger domains with rescaled interface width [37].

We introduce a multiphase-field model following [35] consisting of $N$ phases with corresponding order parameters $\phi=\left\{\phi_{1}, \ldots, \phi_{\alpha}, \ldots, \phi_{N}\right\}^{T}$ and $K$ chemical species with molar fractions $\overline{\boldsymbol{c}}=\left\{\bar{c}_{1}, \ldots, \bar{c}_{i}, \ldots, \bar{c}_{K}\right\}^{T}$. The free-energy functional can be expressed as

$$
\begin{gathered}
\mathcal{F}(\boldsymbol{\phi}, \nabla \boldsymbol{\phi}, \overline{\boldsymbol{c}})=\mathcal{F}_{\text {int }}(\boldsymbol{\phi}, \nabla \boldsymbol{\phi})+\mathcal{F}_{\text {bulk }}(\boldsymbol{\phi}, \overline{\boldsymbol{c}}), \\
\mathcal{F}=\int_{V} f_{\text {grad }}(\boldsymbol{\phi}, \nabla \boldsymbol{\phi})+f_{\text {pot }}(\boldsymbol{\phi})+f_{\text {chem }}(\boldsymbol{\phi}, \overline{\boldsymbol{c}}) d V,
\end{gathered}
$$

consisting of interfacial and bulk contributions. The diffuse interface is determined by two terms; firstly the gradient energy density [46]

$$
f_{\text {grad }}(\nabla \boldsymbol{\phi})=-\varepsilon \sum_{\alpha, \beta>\alpha} \gamma_{\alpha \beta} \nabla \phi_{\alpha} \nabla \phi_{\beta}
$$

with the numerical parameter $\varepsilon$ linked to interface width and the surface energy $\gamma_{\alpha \beta}$ between two phases $\phi_{\alpha}$ and $\phi_{\beta}$. The second term $f_{\text {pot }}$ is the potential energy density. Two common choices for multiphase-field models are the well potential and secondly the obstacle potential, which is often used for numerical performance reasons. We here employ a multiwell approach following [47]

$$
f_{\text {well }}(\phi)=\frac{9}{\varepsilon} \tilde{\gamma}\left(\frac{1}{12}+\frac{1}{2} \sum_{\alpha, \beta>\alpha} \phi_{\alpha}^{2} \phi_{\beta}^{2}+\sum_{\alpha} \frac{\phi_{\alpha}^{4}}{4}-\frac{\phi_{\alpha}^{3}}{3}\right)
$$

with $\tilde{\gamma}=\sum \gamma_{\alpha \beta} \phi_{\alpha}^{2} \phi_{\beta}^{2} / \sum \phi_{\alpha}^{2} \phi_{\beta}^{2}$ and compare it with a multiobstacle potential of the form

$$
f_{\mathrm{ob}}(\boldsymbol{\phi})=\frac{16}{\varepsilon \pi^{2}} \sum_{\alpha, \beta>\alpha}^{N} \gamma_{\alpha \beta} \phi_{\alpha} \phi_{\beta} .
$$

Both approaches in combination with Eq. (10) prevent the nonphysical formation of third phases in binary interfaces [47]. The bulk energy term is expressed as the interpolation of phase-dependent chemical free-energy contributions

$$
f_{\text {chem }}(\boldsymbol{\phi}, \overline{\boldsymbol{c}})=\sum_{\alpha}^{N} f^{\alpha}\left(\boldsymbol{c}^{\alpha}\right) h^{\alpha}(\boldsymbol{\phi}),
$$

where each $f^{\alpha}$ depends on a local phase-dependent composition $c^{\alpha}$ which is given as molar fraction. The mean compositions are defined by interpolation $\bar{c}_{i}=\sum_{\alpha}^{N} c_{i}^{\alpha} h^{\alpha}(\boldsymbol{\phi})$ which follows from interpolation of chemical energies [37] and fulfill the constraint $\sum_{i}^{K} \bar{c}_{i}=1$. The formulation of interpolation functions $h^{\alpha}(\boldsymbol{\phi})$ poses a problem with thermodynamical consistency at multiple junctions. Following [40], we introduce normalized interpolation functions

$$
\begin{aligned}
h^{\alpha}(\boldsymbol{\phi}) & :=\frac{h\left(\phi_{\alpha}\right)}{\sum_{\beta} h\left(\phi_{\beta}\right)}, \\
h_{\mathrm{well}}\left(\phi_{\alpha}\right) & =\phi_{\alpha}^{2}\left(3-2 \phi_{\alpha}\right), \\
h_{\mathrm{ob}}\left(\phi_{\alpha}\right)= & \frac{1}{2}+\frac{2}{\pi}\left[\left(2 \phi_{\alpha}-1\right) \sqrt{\phi_{\alpha}\left(1-\phi_{\alpha}\right)}\right. \\
& \left.+\frac{1}{2} \arcsin \left(2 \phi_{\alpha}-1\right)\right]
\end{aligned}
$$

and adjust our choice to the potential formulation to ensure correct kinetics of the traveling-wave solution in the dualphase case [48]. More detailed discussion of interpolation functions in the multiphase context can be found at [49].

The chemical free energies $f^{\alpha}$ can be fitted by incorporation of CALPHAD data. In this work we use a quadratic fitting for numerical efficiency and furthermore to fulfill the invertibility criterion used for the derivation of the grandpotential model [37]. The chemical energy of each component in phase $\alpha$ is formulated dependent on the phase-dependent mole fraction $c_{i}^{\alpha}$ of species $i$ and three fitting parameters $A_{i}^{\alpha}, B_{i}^{\alpha}$, and $c_{i, \min }^{\alpha}$. The free energy of phase $\alpha$ can then be expressed as the sum over all species contributions

$$
f^{\alpha}\left(c^{\alpha}\right)=\sum_{i}^{K} A_{i}^{\alpha}\left(c_{i}^{\alpha}-c_{i, \min }^{\alpha}\right)^{2}+B_{i}^{\alpha}
$$

The evolution of order parameters can be computed via a variational approach as the summation of dual interactions [46]

$$
\frac{\partial \phi_{\alpha}}{\partial t}=-\frac{1}{\tilde{N} \varepsilon} \sum_{\beta \neq \alpha}^{\tilde{N}} M_{\alpha \beta}\left(\frac{\delta \mathcal{F}}{\delta \phi_{\alpha}}-\frac{\delta \mathcal{F}}{\delta \phi_{\beta}}\right)
$$

where $\tilde{N} \leqslant N$ is the number of active phases and $M_{\alpha \beta}$ denotes the mobility of an $\alpha-\beta$ interface. The variational derivative of 
the overall free energy with respect to $\phi_{\alpha}$ reads

$$
\frac{\delta \mathcal{F}}{\delta \phi_{\alpha}}=\left(\frac{\partial}{\partial \phi_{\alpha}}-\nabla \cdot \frac{\partial}{\partial \nabla \phi_{\alpha}}\right) f(\boldsymbol{\phi}, \nabla \boldsymbol{\phi}, \overline{\boldsymbol{c}}) .
$$

The set of $K-1$ diffusion equations determines the evolution of independent compositions caused by the gradient of phase diffusion potentials $\mu_{j}=\partial f^{\alpha} / \partial c_{j}^{\alpha}$,

$$
\frac{\partial \bar{c}_{i}}{\partial t}=\nabla \cdot\left(\sum_{j=1}^{K-1} \overline{\mathcal{M}}_{i j}(\boldsymbol{\phi}) \nabla \mu_{j}\right) .
$$

The mean mobility comprises the individual phase mobilities $\overline{\mathcal{M}}_{i j}=\sum_{\alpha}^{N} \mathcal{M}_{i j}^{\alpha} h^{\alpha}(\boldsymbol{\phi})$ where each $\mathcal{M}_{i j}^{\alpha}$ is defined by expression $\mathcal{M}_{i j}^{\alpha}=D_{i j}^{\alpha} \partial c_{i}^{\alpha} / \partial \mu_{j}$ in the general case. Inserting Eq. (15) this yields $\mathcal{M}_{i j}^{\alpha}=D_{i j}^{\alpha} \delta_{i j} / 2 A_{i}$. Similar to [38], we solve for phase diffusion potentials instead and rewrite Eq. (18) following [37] to obtain the evolution equation

$$
\begin{aligned}
\frac{\partial \mu_{k}}{\partial t}= & {\left[\sum_{\alpha}^{N} \frac{\partial c_{i}^{\alpha}}{\partial \mu_{k}} h^{\alpha}(\boldsymbol{\phi})\right]^{-1} } \\
& \times\left[\nabla \cdot\left(\sum_{j=1}^{K-1} \overline{\mathcal{M}}_{i j}(\boldsymbol{\phi}) \nabla \mu_{j}\right)-\sum_{\alpha}^{N} c_{i}^{\alpha} \sum_{\beta}^{N} \frac{\partial h^{\alpha}}{\partial \phi_{\beta}} \frac{\partial \phi_{\beta}}{\partial t}\right] .
\end{aligned}
$$

Equations (16) and (19) are two sets of coupled partial differential equations and second order in space.

For the simulations in Sec. IV B a noise term is necessary in order to enable growth of phases that are initially not present. We follow the idea of Schoof et al. [50] and include a term representing thermal fluctuations in the evolution equation

$$
\frac{\partial \phi_{\alpha}}{\partial t}=-\frac{1}{\tilde{N} \varepsilon} \sum_{\beta \neq \alpha}^{\tilde{N}} M_{\alpha \beta}\left(\frac{\delta \mathcal{F}}{\delta \phi_{\alpha}}-\frac{\delta \mathcal{F}}{\delta \phi_{\beta}}\right)+\frac{\partial \zeta}{\partial \phi_{\alpha}}
$$

which is only active in the interface regions as we assume $\mathrm{LiFePO}_{4}$ to nucleate heterogeneously at structural defects such as grain or phase boundaries. The noise is applied as

$$
\zeta=\sum_{\alpha<\beta<\gamma}^{N} A D \phi_{\alpha} \phi_{\beta} \phi_{\gamma}
$$

with an amplitude $A$ and random distribution $D$ which is chosen to be uniform. Simultaneously, correction of the diffusion potentials is included to ensure $\bar{c}$ being unaffected by the noise [51].

\section{MODEL COMPARISON}

In this section we relate the two models presented above in terms of thermodynamical data which is accessible by experiments or first-principles calculations. For better comparability of the modeling approaches, we reformulate the free-energy functionals (1) and (9) and all respective contributions for the case of one diffusing species and constant temperature. This setup is quite simple and yet suitable to investigate phase separating cathode materials with one intercalating species.
All equations are presented in dimensionless form. The CahnHilliard formulation presented in Sec. II A then reduces to

$$
\begin{gathered}
\mathcal{F}^{\mathrm{CH}}(c, \nabla c)=\frac{R T}{V_{\mathrm{m}}} \int_{V} \tilde{f}_{\mathrm{grad}}^{\mathrm{CH}}(\nabla c)+\tilde{f}_{\mathrm{chem}}^{\mathrm{CH}}(c) d V, \\
\tilde{f}_{\mathrm{grad}}^{\mathrm{CH}}(\nabla c)=\tilde{\kappa}|\nabla c|^{2}, \\
\tilde{f}_{\text {chem }, \mathrm{RS}}^{\mathrm{CH}}(c)=c \ln (c)+(1-c) \ln (1-c)+2 \tilde{\chi} c(1-c)
\end{gathered}
$$

which coincides with the models used in many studies of phase separation in single electrode particles $[13,17,19]$ if we neglect mechanical contributions. $\tilde{\kappa}$ and $\tilde{\chi}$ are normalized with respect to the factor $\frac{V_{\mathrm{m}}}{R T}$ and are the only parameters determining interfacial properties and equilibrium compositions. In the following, we will refer to this reduced model as " $\mathrm{CH}$ model"."

Equations (8)-(15) based on nonconservative Allen-Cahn type phase evolution coupled with evolution of the diffusional potential ("AC model") can be simplified in the case of two phases $\alpha, \beta$ with order parameters $\phi_{\alpha}=\phi, \phi_{\beta}=1-\phi$ and one diffusing species with mole fraction $\bar{c}$. The free-energy functional takes the form

$$
\mathcal{F}(\phi, \nabla \phi, \bar{c})=\int_{V} f_{\text {grad }}(\phi, \nabla \phi)+f_{\text {pot }}(\phi)+f_{\text {chem }}(\phi, \bar{c}) d V
$$

with the free-energy density contributions

$$
\begin{gathered}
f_{\text {grad }}(\nabla \phi)=\varepsilon \gamma_{\alpha \beta}|\nabla \phi|^{2}, \\
f_{\text {pot }}(\phi)=\left\{\begin{array}{lc}
\frac{9 \gamma_{\alpha \beta}}{\varepsilon} \phi^{2}(1-\phi)^{2}, & \text { double well } \\
\frac{1 \sigma_{\alpha \beta}}{\varepsilon \pi^{2}} \phi(1-\phi), & \text { double obstacle },
\end{array}\right. \\
f_{\text {chem }}(\phi, \bar{c})=f^{\alpha}(\bar{c}) h(\phi)+f^{\beta}(\bar{c}) h(1-\phi) .
\end{gathered}
$$

The bulk chemical energies for the two phases are given by

$$
\begin{aligned}
& f^{\alpha}\left(c^{\alpha}\right)=A^{\alpha}\left(c^{\alpha}-c_{\min }^{\alpha}\right)^{2}+B^{\alpha}, \\
& f^{\beta}\left(c^{\beta}\right)=A^{\beta}\left(c^{\beta}-c_{\min }^{\beta}\right)^{2}+B^{\beta} .
\end{aligned}
$$

\section{A. Relating conventional parameters}

First of all, the equilibrium composition of the two phases will be related to the model parameters. In the case of the $\mathrm{CH}$ model, they are given by the minima of chemical free energy which can be determined by

$$
\begin{aligned}
\left.\frac{\partial \tilde{f}_{\mathrm{chem}}^{\mathrm{CH}}(c)}{\partial c}\right|_{c=c_{\mathrm{eq}}} & =\ln \left(c_{\mathrm{eq}}\right)-\ln \left(1-c_{\mathrm{eq}}\right)+2 \tilde{\chi}\left(1-2 c_{\mathrm{eq}}\right) \\
& =0 .
\end{aligned}
$$

For $\tilde{\chi}>1$ two minima exist which are symmetric with regard to $c=0.5$. In the AC model the minima of chemical energy density are explicitly introduced as model parameters $c_{\min }^{\alpha}$ and $c_{\min }^{\beta}$. For $B^{\alpha}=B^{\beta}$ they are equivalent to the equilibrium mole fractions. For the case of $A^{\alpha}=A^{\beta}=A$ and a difference in energy levels $B=B^{\alpha}-B^{\beta}$ the equilibrium compositions are 


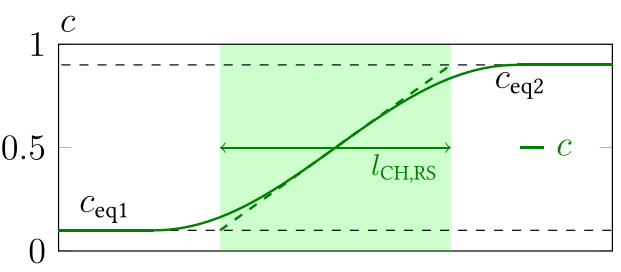

(a) CH-model

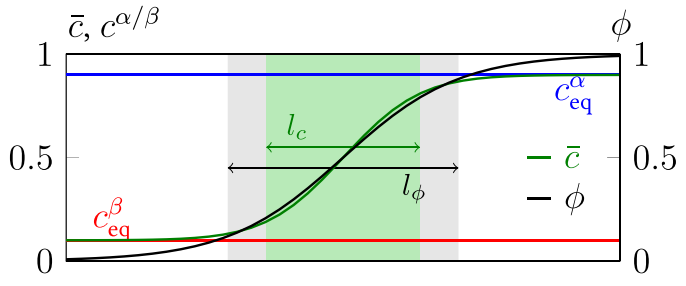

(b) AC-model

FIG. 1. Comparison of the two modeling approaches regarding diffuse interface between two levels of composition in equilibrium state.

shifted from the minima to

$$
\begin{aligned}
& c_{\mathrm{eq}}^{\alpha}=c_{\mathrm{min}}^{\alpha}+\frac{B}{2 A\left(c_{\mathrm{min}}^{\alpha}-c_{\mathrm{min}}^{\beta}\right)}, \\
& c_{\mathrm{eq}}^{\beta}=c_{\min }^{\beta}+\frac{B}{2 A\left(c_{\text {min }}^{\alpha}-c_{\text {min }}^{\beta}\right)} .
\end{aligned}
$$

We now consider a flat interface in equilibrium as shown in Fig. 1 to relate the thermodynamical properties interfacial energy $\sigma$ and interface width $l$. We therefore start with calculation of the respective values in the Cahn-Hilliard case. Following [43], the stationary solution of concentration that minimizes the functional (22) holds condition $\tilde{f}_{\text {chem, RS }}^{\mathrm{CH}}(c)-$ $f_{\text {min }}=\tilde{\kappa}\left(\frac{d c}{d x}\right)^{2}$ with $f_{\text {min }}=\tilde{f}_{\text {chem }, \mathrm{RS}}^{\mathrm{CH}}\left(c_{\mathrm{eq}}\right)$ being the minimal chemical energy. Therefore, the interfacial energy can be expressed as

$$
\begin{aligned}
\sigma & =2 \frac{R T}{V_{\mathrm{m}}} \int_{-\infty}^{\infty} \tilde{f}_{\text {chem }, \mathrm{RS}}^{\mathrm{CH}}(c)-f_{\mathrm{min}} d x \\
& =2 \frac{R T}{V_{\mathrm{m}}} \int_{c_{\mathrm{eq} 1}}^{c_{\mathrm{eq} 2}} \sqrt{\tilde{\kappa}\left[\tilde{f}_{\mathrm{chem}, \mathrm{RS}}^{\mathrm{CH}}(c)-f_{\mathrm{min}}\right]} d c .
\end{aligned}
$$

The interface width $l$ is approximated by the distance between intersections of a linear tangent in $c=0.5$ and the equilibrium compositions

$$
\begin{aligned}
l_{\mathrm{CH}, \mathrm{RS}} & =\left.\frac{\left(c_{\mathrm{eq} 2}-c_{\mathrm{eq} 1}\right)}{\frac{d c}{d x}}\right|_{c=0.5} \\
& =\left(c_{\mathrm{eq} 2}-c_{\mathrm{eq} 1}\right) \sqrt{\frac{\tilde{\kappa}}{\tilde{f}_{\mathrm{chem}, \mathrm{RS}}(c=0.5)}} .
\end{aligned}
$$

The tangent construction and computed interface width are indicated by green shading in Fig. 1(a). Note that interfacial energy and interface width are intrinsically coupled by the choice of $\tilde{\kappa}$ for the $\mathrm{CH}$ model.

In the AC model interface and bulk contributions are decoupled, which becomes obvious upon consideration of an interface in equilibrium as the one depicted in Fig. 1(b). The chemical energy density contribution (29) vanishes and the interface properties are solely determined by Eqs. (26) and (27). The resulting equilibrium profile of a two-phase flat interface for the double-well potential $\phi(x)=\frac{1}{2}+\frac{1}{2} \tanh \left(\frac{3}{2 \varepsilon} x\right)$ corresponds to an interfacial energy of $\sigma=\gamma_{\alpha \beta}$ and the interface width $l_{\phi}^{\mathrm{DW}}=4 \varepsilon / 3$ for linear tangent fit of the gradient in $\phi=$ 0.5 . Similarly, the choice of double-obstacle potential yields $\phi(x)=\frac{1}{2}+\frac{1}{2} \sin \left(\frac{4}{\varepsilon \pi} x\right)$ with an interfacial energy $\sigma=\gamma_{\alpha \beta}$ and the interface width $l_{\phi}^{\mathrm{DO}}=\varepsilon \pi / 2$. The profile of mean composition follows by interpolation $\bar{c}=c_{\mathrm{eq}}^{\alpha} h(\phi)+c_{\mathrm{eq}}^{\beta}[1-h(\phi)]$.
The AC model features the advantage that surface energy $\gamma_{\alpha \beta}$ and interface width $l(\varepsilon)$ are two independent variables.

\section{B. Stability analysis}

This section is dedicated to the examination of stability regarding spontaneous formation of new phases as in the case of spinodal decomposition. The stability of the $\mathrm{CH}$ model has been studied in the works of Cahn and Hilliard [52] and by Cahn $[53,54]$. Starting with a constant initial composition in the whole domain, the stability of the system toward small pertubations can be analyzed. Stable behavior would lead to a decay of small pertubations while in the unstable region any small pertubation will lead to spinodal decomposition. The spinodal is defined by vanishing second derivative of free-energy density where the critical radius for a nucleus becomes zero [53], which yields

$$
\frac{\partial^{2} \tilde{f}_{\mathrm{chem}, \mathrm{RS}}^{\mathrm{CH}}(c)}{\partial c^{2}}=\frac{1}{c(1-c)}-4 \tilde{\chi}=0
$$

under the assumption of negligible gradients $|\nabla c| \ll 1$. For $\tilde{\chi} \leqslant 1$ only one solution $c_{\text {crit }}=0.5$, for values $\tilde{\chi}>1$ two solutions are existent, and spinodal decomposition occurs for all concentrations within the interval $c \in\left(c_{\text {crit, } 1}, c_{\text {crit }, 2}\right)$. Equation (24) is plotted in Fig. 2 for a specific choice of parameter $\chi>1$ with distinct spinodal region.

The simulation of spontaneous phase separation has long been considered impossible within the framework of grandpotential-based phase-field models [36] but has recently been accomplished by Aagesen et al. [42]. In this study, we extend similar treatment to the multiphase-field approach. To derive a criterion for critical composition, we first express Eq. (28) explicitly as a function of average composition. The

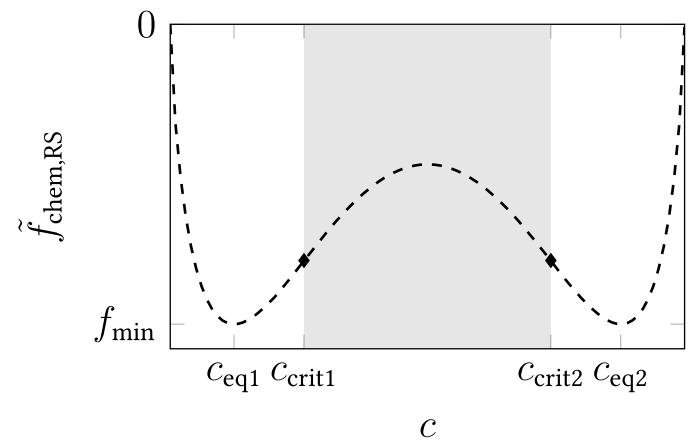

FIG. 2. Regular solution free-energy density $\tilde{f}_{\text {chem,RS }}$ for $\tilde{\chi}>1$. The spinodal points are indicated by black marks and the spinodal region by gray shading. 
phase-dependent mole fractions in the diffuse interface are linked by the assumption of equal phase diffusion potentials for all phases $\mu=\mu^{\alpha}=\mu^{\beta}[55]$ which yields

$$
\mu=\frac{\partial f^{\alpha}}{\partial c^{\alpha}}=\frac{\partial f^{\beta}}{\partial c^{\beta}}=\frac{\partial f_{\text {chem }}}{\partial \bar{c}} .
$$

From Eqs. (29) and (35) we get the equality $2 A^{\alpha}\left(c^{\alpha}-c_{\min }^{\alpha}\right)=$ $2 A^{\beta}\left(c^{\beta}-c_{\min }^{\beta}\right)$. Furthermore, applying the relation $\bar{c}=$ $c^{\alpha} h(\phi)+c^{\beta} h(1-\phi)$ for the mean concentration which can be derived from the interpolation of chemical energy densities (13) [37], we express the phase-dependent chemical energy density Eq. (29) in terms of variables $\bar{c}, \phi$ :

$$
f^{\alpha}(\bar{c}, \phi)=A^{\alpha}\left(\frac{\bar{c}-\left[c_{\min }^{\alpha} h(\phi)+c_{\min }^{\beta} h(1-\phi)\right]}{h(\phi)+\frac{A^{\alpha}}{A^{\beta}} h(1-\phi)}\right)^{2}+B^{\alpha} .
$$

By inserting Eq. (36) into Eq. (28) and using the symmetry of interpolation function $h(1-\phi)=1-h(\phi)$, the free-energy density is expressed as

$$
\begin{aligned}
f_{\text {chem }}(\bar{c}, \phi)= & \frac{A^{\alpha} A^{\beta}\left(\bar{c}-\left\{c_{\min }^{\alpha} h(\phi)+c_{\min }^{\beta}[1-h(\phi)]\right\}\right)^{2}}{A^{\beta} h(\phi)+A^{\alpha}[1-h(\phi)]} \\
& +\left(B^{\alpha}-B^{\beta}\right) h(\phi)+B^{\beta} .
\end{aligned}
$$

Without loss of generality, we set the reference of energy level to $B^{\beta}=0$ and rename $B^{\alpha}=B$. By introducing the term $\bar{c}_{\text {min }}(\phi)=\left\{c_{\text {min }}^{\alpha} h(\phi)+c_{\text {min }}^{\beta}[1-h(\phi)]\right\}$ and assuming a simplified system with $A^{\alpha}=A^{\beta}=A$ the free-energy density takes the form

$$
f_{\text {chem }}(\bar{c}, \phi)=A\left[\bar{c}-\bar{c}_{\text {min }}(\phi)\right]^{2}+B h(\phi) .
$$

To investigate the stability, we first need to examine the stationary solutions of the given system of evolution equations in the two-phase case

$$
\varepsilon \dot{\phi}=-M\left(\frac{\delta \mathcal{F}}{\delta \phi}\right), \quad \frac{\partial \bar{c}}{\partial t}=\nabla \cdot\left[D(\phi) \nabla\left(\frac{\delta \mathcal{F}}{\delta \bar{c}}\right)\right] .
$$

The coupled system of equations is then given by

$$
\left[\begin{array}{c}
\dot{\phi} \\
\dot{\bar{c}}
\end{array}\right]=\left[\begin{array}{c}
\frac{M}{\varepsilon}\left(2 \varepsilon \gamma_{\alpha \beta} \nabla^{2} \phi-\frac{\partial f_{\mathrm{pot}}}{\partial \phi}+\left\{2 A \Delta c_{\min }\left[\bar{c}-\bar{c}_{\min }(\phi)\right]-B\right\} \frac{\partial h}{\partial \phi}\right) \\
D\left(\nabla^{2} \bar{c}-\Delta c_{\min } \frac{\partial h}{\partial \phi} \nabla^{2} \phi-\Delta c_{\min } \frac{\partial^{2} h}{\partial \phi^{2}}(\nabla \phi)^{2}\right)
\end{array}\right]
$$

with $\Delta c_{\min }=c_{\min }^{\alpha}-c_{\min }^{\beta}$. Examination of stationary solutions $[\dot{\phi}, \dot{\bar{c}}]^{T}=0$ for $\nabla \phi \approx 0$ and thus $\phi$ constant in the whole domain, yields for the double-well potential

$$
\begin{aligned}
& -\frac{18 \gamma_{\alpha \beta}}{\varepsilon} \phi(1-\phi)(1-2 \phi) \\
& \quad+\left\{2 A \Delta c_{\min }\left[\bar{c}-\bar{c}_{\min }(\phi)\right]-B\right\} \frac{\partial h}{\partial \phi} \stackrel{!}{=} 0
\end{aligned}
$$

with $\partial h / \partial \phi=6 \phi(1-\phi)$, which results in

$$
\begin{gathered}
\bar{c}_{\text {stat }}=c_{0} \text { (const.), } \phi=0 \text { or } \phi=1, \\
\bar{c}_{\text {stat }}=c_{\text {min }}^{\beta}+\Delta c_{\min } \phi^{2}(3-2 \phi) \\
+\frac{B}{2 A \Delta c_{\text {min }}}+\frac{3 \gamma_{\alpha \beta}}{2 A \varepsilon \Delta c_{\min }}(1-2 \phi), \phi \in(0,1) .
\end{gathered}
$$

Stability analysis of these stationary solutions (see Appendix A) shows that the critical solution for $\phi \in(0,1)$ is always unstable, whereas in the case of $\phi=0$ and $\phi=1$ a change in the stability behavior can be observed. The domain of instability is limited by the critical composition

$$
\begin{aligned}
& c_{\text {crit }}(\phi=0)=c_{\mathrm{eq}}^{\beta}+\frac{18 \gamma_{\alpha \beta}}{12 A \Delta c_{\min } \varepsilon}, \\
& c_{\text {crit }}(\phi=1)=c_{\mathrm{eq}}^{\alpha}-\frac{18 \gamma_{\alpha \beta}}{12 A \Delta c_{\min } \varepsilon} .
\end{aligned}
$$

These two critical values are symmetric with regard to $\bar{c}=$ $0.5\left(c_{\mathrm{eq}}^{\alpha}+c_{\mathrm{eq}}^{\beta}\right)$, which is due to the choice of $A^{\alpha}=A^{\beta}=A$. The difference in energy height $B$ results in a constant shift of critical concentrations analogous to the shift of equilibrium compositions Eq. (31) according to the common tangent construction. For the obstacle potential, examination of the stationary solutions of Eq. (40) leads to the criterion

$$
\begin{aligned}
& -\frac{16 \gamma_{\alpha \beta}}{\varepsilon \pi^{2}}(1-2 \phi) \\
& \quad+\frac{8}{\pi} \sqrt{\phi(1-\phi)}\left\{2 A \Delta c_{\min }\left[\bar{c}-\bar{c}_{\min }(\phi)\right]-B\right\} \stackrel{!}{=} 0,
\end{aligned}
$$

which results in

$$
\begin{aligned}
\bar{c}_{\mathrm{stat}}= & c_{\min }^{\beta}+\Delta c_{\min } h_{\mathrm{ob}}(\phi) \\
& +\frac{B}{2 A \Delta c_{\min }}+\frac{\gamma_{\alpha \beta}}{A \varepsilon \pi \Delta c_{\min }} \frac{1-2 \phi}{\sqrt{\phi(1-\phi)}},
\end{aligned}
$$

which is unstable for all $\phi \in(0,1)$. In the following section we study the numerical behavior of the two models to investigate the validity of theoretical predictions.

\section{SIMULATION RESULTS AND DISCUSSION}

\section{A. Phase separation in a two-phase model system}

The first study aims at proving the theoretical predictions in Sec. III B and comparing the simulation results. In Table I, a set of nondimensionalized simulation parameters is shown

TABLE I. Input parameters for model validation.

\begin{tabular}{lcc}
\hline \hline Parameter & Symbol & Value \\
\hline Equilibrium mole fractions & $c_{\mathrm{eq}}^{\alpha}$ & 0.9 \\
& $c_{\mathrm{eq}}^{\beta}$ & 0.1 \\
Interfacial energy & $\sigma$ & 0.5 \\
Diffusivity & $D$ & 1.0 \\
\hline \hline
\end{tabular}



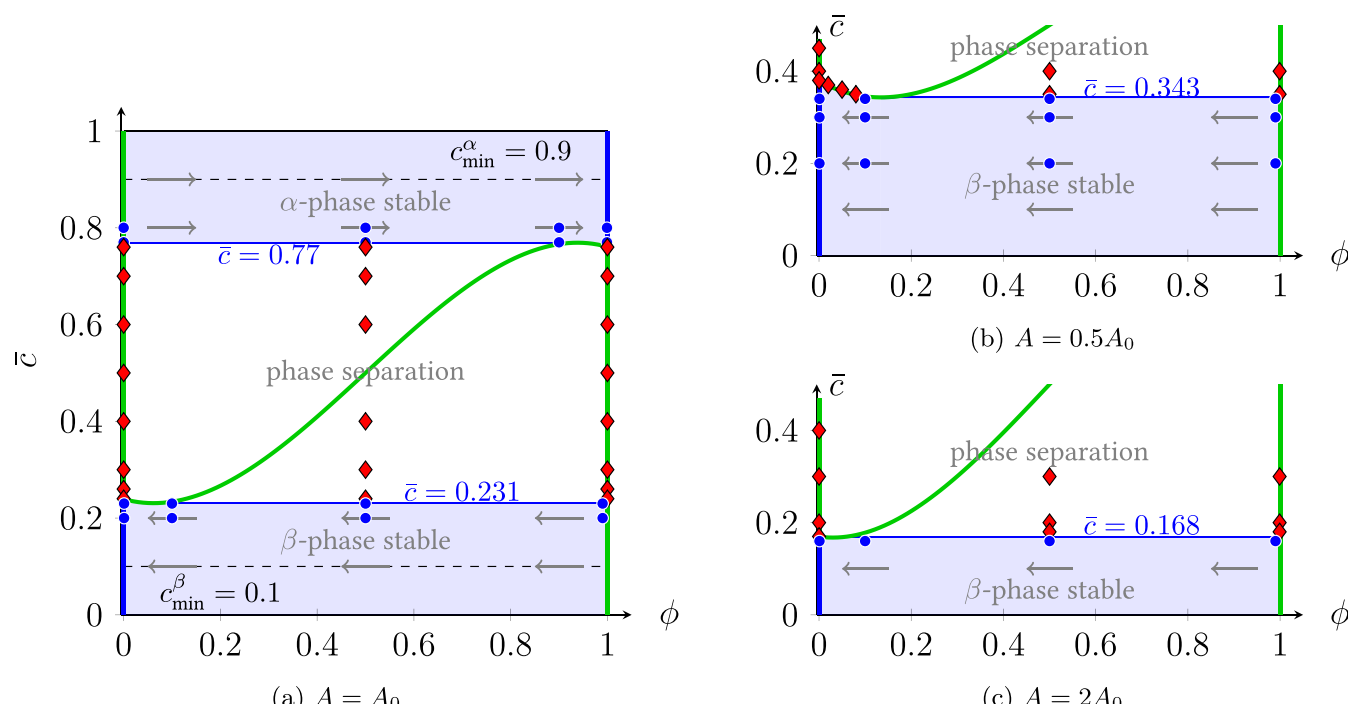

(b) $A=0.5 A_{0}$

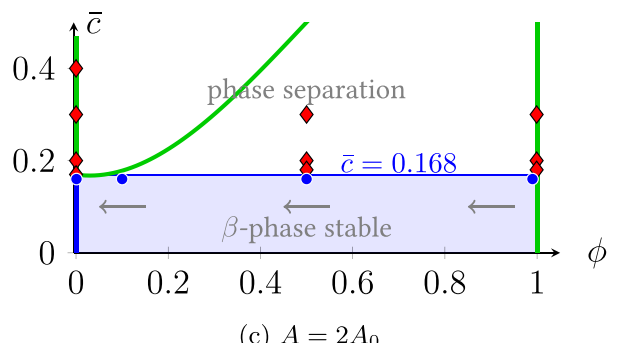

FIG. 3. Stability map for well potential in the variable space $\phi \in[0,1]$ and $\bar{c} \in[0,1]$. Stationary solutions according to Eqs. (42) and (43) are shown in blue if stable and green for the case of instability. All simulations marked by blue dots feature decaying fluctuations and one stable phase, while red diamonds indicate simulations exhibiting phase separation.

which corresponds to a choice of $\tilde{\chi}=1.373$ and $\tilde{\kappa}=2.854$ for the $\mathrm{CH}$ model. The combination of both yields an interface width according to Eq. (33) of $l_{\mathrm{CH}, \mathrm{RS}}=5.058$. For the AC model we choose $\gamma_{\alpha \beta}=0.5$ to fit the surface energy and request equal interface width in accordance with the $\mathrm{CH}$ model. As shown in Fig. 1(b), the interfacial widths of the concentration and the phase profile are not identical in the general case of an arbitrary interpolation function $h(\phi)$. The equilibrium mole fractions are fitted by choice of $c_{\min }^{\alpha}=$ $0.9, c_{\min }^{\beta}=0.1$, and $B=0$. The choice of $\tilde{\chi}$ sets the spinodal compositions $c_{\text {crit, } 1}$ and $c_{\text {crit, } 2}$ which are fitted by criterion (44) to $A^{\alpha}=A^{\beta}=6.73 / \varepsilon$ such that $c_{\text {crit }}(\phi=0)=c_{\text {crit }, 1}$. Because of symmetry, $c_{\text {crit }}(\phi=1)=c_{\text {crit }, 2}$ is also fulfilled.

The predicted instability (44) is studied for different initial configurations in the variable space $\phi \in[0,1]$ and $\bar{c} \in[0,1]$. Figure 3 shows the results for the double-well potential and three different choices of $A^{\alpha}=A^{\beta}$ while all other parameters are kept constant. For all cases, three domains with different phase evolution can be identified. All setups with an initial composition above the upper critical one lead to decaying concentration fluctuations and stable $\alpha$ phase. The phase values in the whole domain trend toward $\phi=1$, as indicated by the gray arrows. The second stable domain below the lower critical composition favors the $\beta$ phase and $\phi$ values trend toward zero. The area in between is characterized by unstable amplification of small fluctuations and the formation of regular homogeneous two-phase patterns as in the example shown in Fig. 4. All simulations are in agreement with the theoretical predictions.

We apply the same procedure to study the stability for the obstacle potential. The results are shown in Fig. 5 and once again agreement with the theoretical predictions is observed. The same three domains as before can be identified and are separated by the stationary solution Eq. (47). A qualitative difference to the well potential is the nonexistence of a stable stationary solution for $\phi=0$ or $\phi=1$ due to the shape of the potential. We nevertheless observe stable $\alpha$ or $\beta$ phase within the blue shaded areas as the energy exhibits minimal values at the boundary and $\phi$ is limited to $\phi \in[0,1]$ by the Gibbs simplex constraint.

The resulting pattern formation during the spinodal decomposition process is highly dependent on the choice of energy density contributions. In Fig. 6, the demixing process is compared for the three modeling approaches. The parameters were chosen according to Table I. The kinetics of the systems are comparable while the structures of the emerging phase are slightly different. While the $\mathrm{CH}$ model tends to form spheroidal precipitates, the AC model and especially the obstable potential formulation form more entangled structures.

\section{B. Multigrain intercalation}

As pointed out in the Introduction, LFP has been well studied over the past several years employing the phase-field method. To prove applicability of the newly proposed modeling approach via comparison of the $\mathrm{CH}$ model with the $\mathrm{AC}$ model, we choose a rather simplified 1D particle intercalation setup neglecting mechanical contributions and applying constant flux as a boundary condition (BC), as shown in Fig. 7(a). This BC reflects discharge at constant current (CC). We furthermore study the multiparticle interaction upon discharge at $C$ rate $C=1$ with the 2D setup shown in Fig. 7(b).

TABLE II. Material input data for LFP.

\begin{tabular}{lccc}
\hline \hline Parameter & Symbol & Value & Ref. \\
\hline Energy coefficient & $\tilde{\chi}$ & 2.4055 & {$[10]$} \\
Gradient energy & $\tilde{\kappa}$ & $1.475 \times 10^{-18} \mathrm{~m}^{2}$ & {$[11]$} \\
Diffusion coefficient & $D_{0}$ & $10^{-15} \mathrm{~m}^{2} / \mathrm{s}$ & {$[11]$} \\
Molar volume & $V_{\mathrm{m}}$ & $4.38 \times 10^{-5} \mathrm{~m}^{3} / \mathrm{mol}$ & {$[10]$} \\
\hline \hline
\end{tabular}




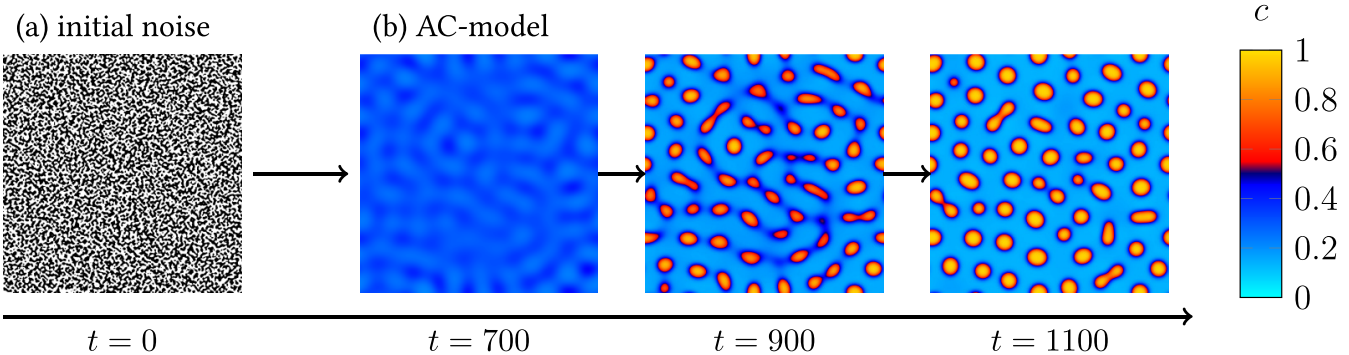

FIG. 4. Concentration evolution due to spinodal decomposition in 2D domain with $200 \times 200$ cells starting from (a) initial noise where cells with composition above $c=0.3$ are colored in black. Three time steps of temporal evolution are shown for (b) AC model with double-well potential, $\varepsilon=4.8$ and $M=10$.

LFP exhibits strongly anisotropic diffusion [12] and anisotropic surface energy properties [27]. The influence of diffusion anisotropy is reduced by antisite defects, which is why we assume homogeneous diffusion and, therefore, employ the parameters used in [11] which are partly taken from [10]. An overview of the parameters is given in Table II. The length of the simulation domain is chosen to be $L=300 \mathrm{~nm}$ and discretized with 300 cells. The influence of particle size on the miscibility gap is considered negligible as the resulting interfacial width for parameters in Table II is $1.6 \mathrm{~nm}$. We choose $C$ rate $C=0.1$ as a reference and start with an initial composition of $\bar{c}=0.01$. From these values and the ones given in Table II, the constant boundary flux $J_{N}=L C / V_{\mathrm{m}}=$ $3.17 \times 10^{-5} \mathrm{~mol} / \mathrm{m}^{2} \mathrm{~s}$ can be determined which is used as a boundary condition on the left side of the simulation domain. No flux boundary condition $\nabla \bar{c}=0$ is applied on the right-hand side. All simulations are carried out for constant temperature $T=300 \mathrm{~K}$. Figure 8(a) shows the simulation result employing the $\mathrm{CH}$ model, which is used as a baseline for comparison. The discretization is sufficient to resolve the phase boundary between the FP and LFP phases. Coarser grid resolution results in large deviation of the chemical energies of the system due to numerical errors, while finer spatial resolution results in minor improvement in accuracy, but with the disadvantage of a tremendous increase in computational cost. After approximately $1 \mathrm{~h}$, phase separation occurs and the phase front moves through the electrode. After $10 \mathrm{~h}$, the particle is fully charged. The parameters given in Table II correspond to a spinodal mole fraction of $c_{\text {crit, } 1}=0.118$ and interface energy $\sigma=72 \frac{\mathrm{mJ}}{\mathrm{m}^{2}}$. These values are fitted for the AC model following the procedure described in Secs. III A and III B. Comparison with the AC model shows that the position of the phase front is reproduced accurately even when interfacial width is increased and the number of grid points is reduced. However, we note that there is a qualitative difference between the two modeling approaches. The $\mathrm{CH}$ model allows for nucleation in the composition range between the first local minimum and the spinodal composition if fluctuations are large enough to reach instability (the mechanism of nucleation continuously converts into spinodal phase separation [53]). On the other hand, it can be derived from the stability maps Figs. 3 and 5 that no growth of the second phase is possible in the domain below a certain critical composition with the AC model. Accordingly, phase separation occurs at

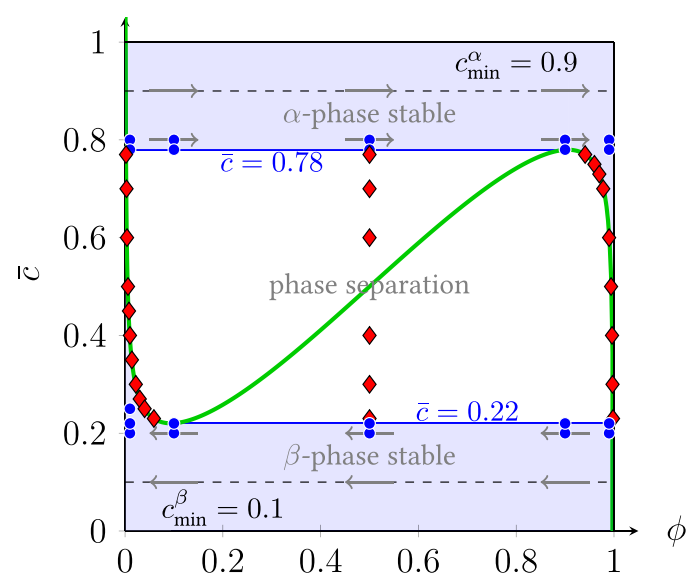

(a) $A=A_{0}$

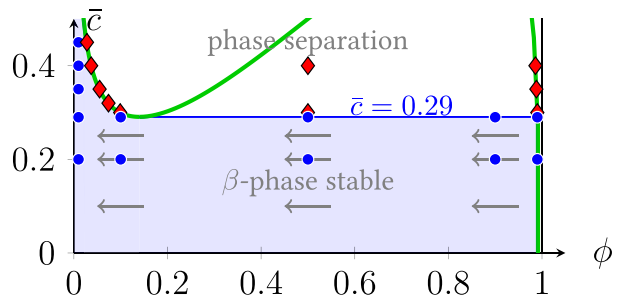

(b) $A=0.5 A_{0}$

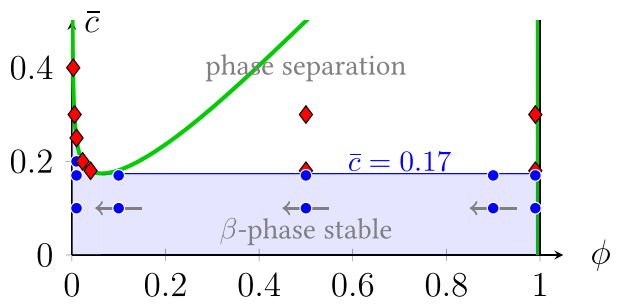

(c) $A=2 A_{0}$

FIG. 5. Stability map for obstacle potential. Stationary solutions according to Eq. (47) are always unstable and therefore shown in green. All simulations marked by blue dots feature decaying fluctuations and one stable phase while red diamonds indicate simulations exhibiting phase separation. 
(a)

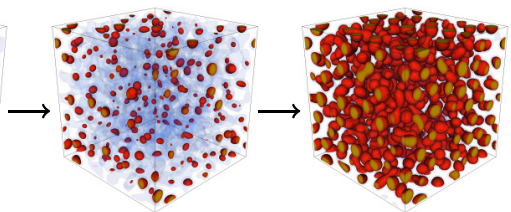

(b)

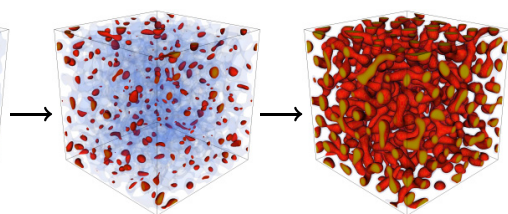

(c)

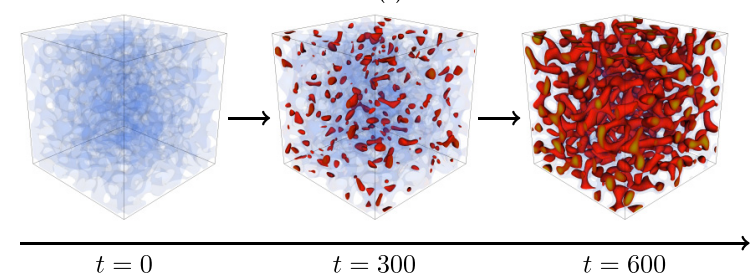

FIG. 6. Concentration evolution in a three-dimensional domain with $200 \times 200 \times 200$ voxels and initial $\bar{c}=0.3$. Opacity of composition below $\bar{c}=0.5$ is set to $20 \%$ for better visibility of the phase separation. Three time steps of temporal evolution are shown for (a) $\mathrm{CH}$ model, (b) AC model with well potential, and (c) AC model with obstacle potential.

$1 \mathrm{~h}$ in the $\mathrm{CH}$ model shortly before the spinodal concentration is reached in the system due to the fluctuation induced by the boundary condition while the AC model still exhibits one phase and decomposes after $1.1 \mathrm{~h}$ of charge time.

The computation time can be massively reduced by increasing $\varepsilon$ and reducing the discretization points as the stable time step $\Delta t$ is linked to spatial discretization via the Courant-Friedrichs-Lewy (CFL) condition for finite difference schemes. Comparison of some simulations in Fig. 8(b)

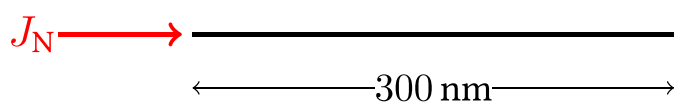

(a) $1 \mathrm{D}$ electrode intercalation

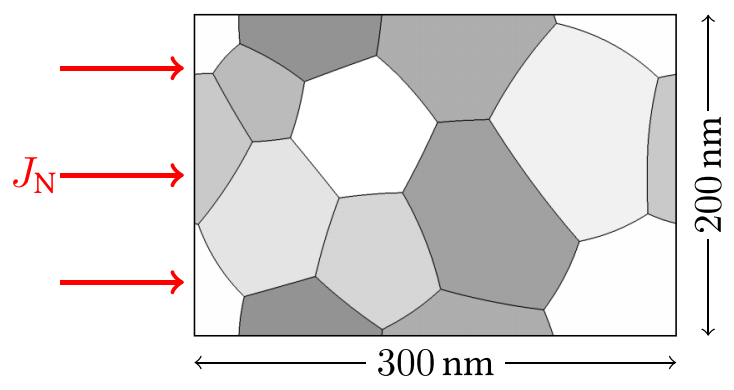

(b) 2D multi-particle intercalation

FIG. 7. Simulation setup for particle intercalation study.

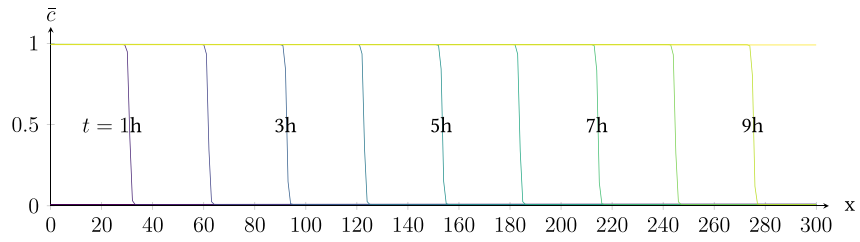

(a)

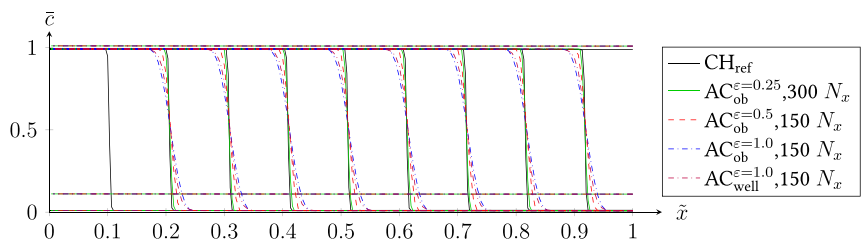

(b)

FIG. 8. Simulation results of 1D particle charge. (a) Temporal evolution of $\bar{c}$ with $\mathrm{CH}$-model, $N_{x}=300$. (b) Comparison of $\mathrm{CH}$ model and AC-model simulations for varying $\varepsilon$.

yields the results shown in Table. III. The value $\Delta t_{\max }$ refers to the maximal possible time step that yields a numerically stable simulation employing a temporal discretization via the explicit Euler method with constant time stepping. It strongly influences the absolute computational time. The $\mathrm{CH}$ model exhibits a relatively long wall time due to the massive amount of simulation steps and the strong dependence of $\Delta t$ on $\Delta x$ due to the fourth-order PDE. This simulation is taken as a reference for the relative time. The corresponding simulation with $N_{x}=300$ employing the AC model features a slightly larger stable time step and therefore, computational time is reduced by $4 \%$. Increasing the interfacial width enables a reduction of grid points $N_{x}$ in the AC model while the $\mathrm{CH}$ model formulation does not allow for rescaling. Coarser spatial discretization would lead to a large increase of numerical errors. In this example, computation time could be reduced by a factor of 8.2 by applying rescaling of the numerical interface width and reduction of grid points. The choice of obstacle potential, in particular, reduces computational cost because of its finite interface width. This effect becomes even more pronounced in two- or three-dimensional simulations where the total amount of cells scales with $(\Delta x)^{2}$ and $(\Delta x)^{3}$, respectively.

In our last example we study the intercalation behavior of a multigrain section. For performance reasons, we only choose the obstacle potential. We start from a Voronoi filling

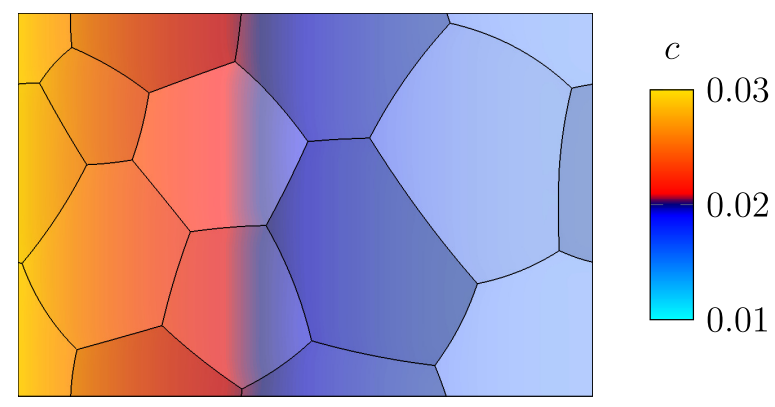

FIG. 9. Composition gradient within a multigrain cathode section under $\mathrm{CC}$ discharge at $t=0.01 \mathrm{~h}$. 
TABLE III. Computation time.

\begin{tabular}{|c|c|c|c|c|c|c|}
\hline \multirow[b]{2}{*}{ Simulation } & \multirow[b]{2}{*}{$N_{x}$} & \multirow[b]{2}{*}{$\Delta t_{\max }$} & \multicolumn{2}{|c|}{$1 \mathrm{CPU}$} & \multicolumn{2}{|c|}{5 CPUs } \\
\hline & & & Wall time & Rel. time & Wall time & Rel. time \\
\hline $\mathrm{CH}$ model & 300 & $7.5 \times 10^{-8} \mathrm{~s}$ & $8231.7 \mathrm{~s}$ & $100 \%$ & $3012.0 \mathrm{~s}$ & $100 \%$ \\
\hline $\mathrm{AC}$ model $\mathrm{AC}_{\mathrm{ob}}^{\varepsilon=0.25}$ & 300 & $1.0 \times 10^{-7} \mathrm{~s}$ & $7921.6 \mathrm{~s}$ & $96.2 \%$ & $2893.7 \mathrm{~s}$ & $96.1 \%$ \\
\hline AC model $\mathrm{AC}_{\text {well }}^{\varepsilon=1.0}$ & 150 & $5.0 \times 10^{-7} \mathrm{~s}$ & $2493.6 \mathrm{~s}$ & $30.3 \%$ & $725.5 \mathrm{~s}$ & $24.1 \%$ \\
\hline AC model $\mathrm{AC}_{\mathrm{ob}}^{\varepsilon=1.0}$ & 150 & $5.0 \times 10^{-7} \mathrm{~s}$ & $1003.0 \mathrm{~s}$ & $12.2 \%$ & $479.3 \mathrm{~s}$ & $15.9 \%$ \\
\hline
\end{tabular}

of ten phases which have undergone some volume preserving relaxation to reduce the initially high interfacial energies and reproduce equilibrium angles at triple junctions. Phase pairs that are assumed to have the same interfacial energy $\gamma_{\alpha \beta}=$ $72 \frac{\mathrm{mJ}}{\mathrm{m}^{2}}$ and the same parameters according to Table II are used. We furthermore apply a boundary flux corresponding to $1 C$ discharge rate at the left-hand side and no flux $\mathrm{BC}$ at the right side of the simulation domain. The phase boundary between FP and LFP is often assumed to be coherent [8] while grain boundaries are noncoherent. This is reflected in the choice of corresponding LFP phases that can only grow at the expense of an FP grain, hence preserving the original grain boundaries.

In Fig. 9, the lithium composition is shown at the beginning of the intercalation process at $t=0.01 \mathrm{~h}$. Composition gradually decays in horizontal direction and the gradient is only weakly influenced by the grain boundaries. Consideration of anisotropic diffusion or grain boundary diffusion would most likely largely influence the transport but are neglected at this point. These are subject to future studies. Phase separation is initiated at lower composition $\bar{c}$ than in the previous example as the presence of equilibrium interfaces and multiple junctions lowers the barrier toward nucleation of the LFP phase. The considerations presented in Appendix B confirm this observation analytically. Using Eq. (47), we fit the local minimum of $\bar{c}_{\text {stat }}$ (see Fig. 5) to $\bar{c}_{\text {stat,min }}^{\text {dual }}=c_{\text {crit }, 1}=0.118$. The resulting choice of parameters can be inserted into Eq. (B4) and analysis shows that the critical composition for nucleation of the LFP phase within an equilibrium interface of two FP phases is reduced to $\bar{c}_{\text {stat,min }}^{\text {triple }}=0.0725$. The stability analysis furthermore reveals that Eq. (B4) is indeed unstable with regard to small fluctuations as the criterion Eq. (B7) is not fulfilled for all $\left\{\phi_{\alpha}, \phi_{\beta}, \phi_{\gamma}\right\}$ on the Gibbs simplex $\sum_{\alpha} \phi_{\alpha}=1$. Thus, phase separation occurs for compositions higher than $\bar{c}_{\text {stat,min }}^{\text {triple }}$ in a two-phase equilibrium interface. Higher order junctions most likely lower the nucleation barrier additionally but proving this analytically was out of the scope of this paper. We note, however, that in the present simulations nucleation of the LFP phases was always initiated at higher order junctions, which might be due to the fact that less surface is created. These considerations strongly support the choice of the noise term Eq. (21), which is only active in the interfacial regions based on the assumption of heterogeneous nucleation.

Once the critical composition has been exceeded and phase separation occurs, grain-by-grain-like filling can be observed. Figure 10(a) shows two grains in which nucleation has been initiated and LFP phases grow on the expense of the corresponding FP phase. The phase transformation within each grain is triggered at higher order junctions such as that marked by the red circle in Fig. 10(b), and then proceeds through the grain. If multiple nucleation events happen simultaneously, the energetically more favorable grain will be filled completely by diffusion of ions from the surrounding grains before the next grain will be filled (see Supplemental Material [56]). This illustrates the strong coupling between diffusion and phase transformation. Over time, all grains within the section exhibit phase transformation and a front between LFP phases and FP phases moves through the simulation domain as shown in Fig. 11. Neighboring grains influence the progression of the phase transformation as it is energetically favorable for the intercalation to proceed in a grain-by-grain process. This seems reasonable from a physical point of view as the overall interfacial energy is reduced. From Fig. 11 and the Supplemental Material, we conclude that the evolving LFP phases form $90^{\circ}$ contact angles at the left and right boundaries of the domain due to the $\nabla \bar{c}=0 \mathrm{BC}$, but also at the preexistent internal grain boundaries. This is in agreement with the Young

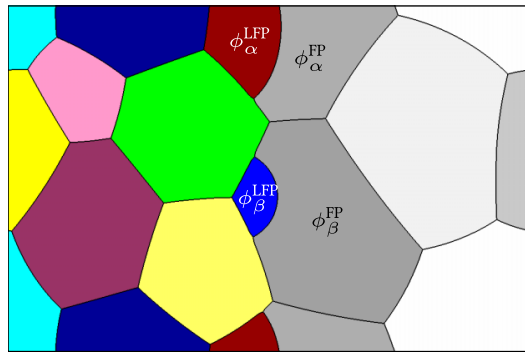

(a) multiphase-field $\phi$

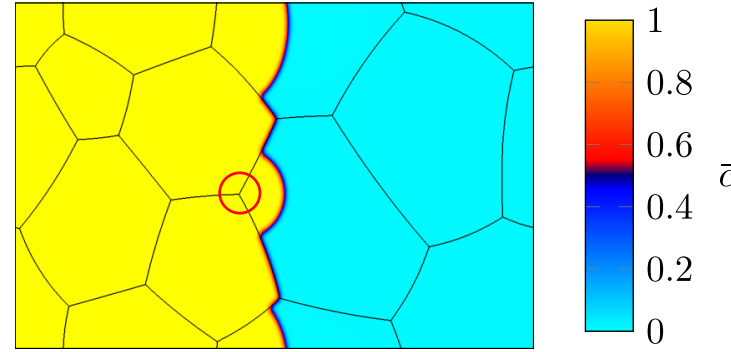

(b) composition $\bar{c}$

FIG. 10. Intercalation in a 2D multigrain cathode section with $200 \times 300$ cells under constant current discharge. Evolution of phases (a) is coupled to composition and (b) is displayed here for $1 C$ rate at time $t=0.5 \mathrm{~h}$. 


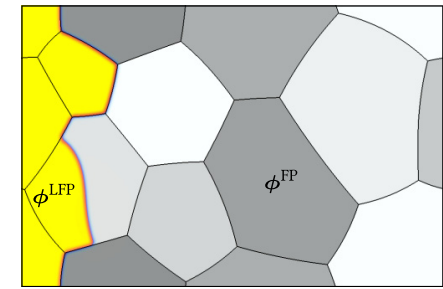

(a) $t=0.15 \mathrm{~h}$

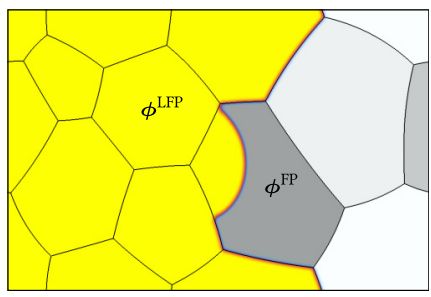

(d) $t=0.60 \mathrm{~h}$

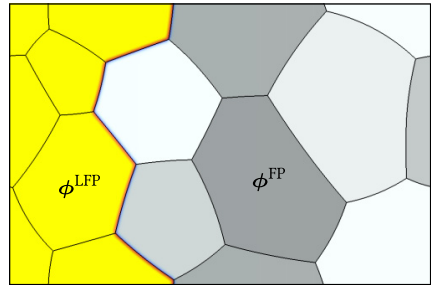

(b) $t=0.30 \mathrm{~h}$

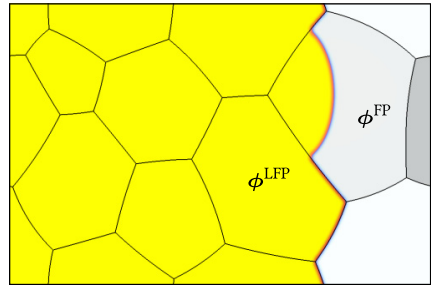

(e) $t=0.75 \mathrm{~h}$

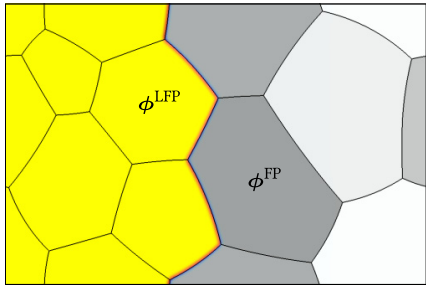

(c) $t=0.45 \mathrm{~h}$

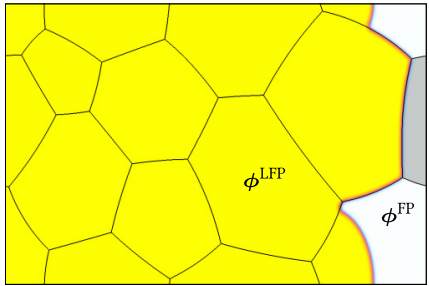

(f) $t=0.90 \mathrm{~h}$

FIG. 11. Evolution of FP to LFP phase transformation during intercalation in 2D multigrain cathode section with $200 \times 300$ cells under CC discharge with $1 C$ rate.

equation for the wetting angle on a rigid surface where all three phase pairs exhibit the same surface energy $\gamma_{\alpha \beta}$. In this sense, the initial grain boundaries can be interpreted as a rigid substrate because of their immobility. The observed phase front between the LFP and FP phase within each grain then evolves such that curvature and total surface are minimized while keeping $90^{\circ}$ contact angles with the grain boundaries. Movies of the intercalation process are available in the Supplemental Material [56].

\section{CONCLUSION}

In this work, we presented a computationally efficient alternative to model intercalation in cathode materials exhibiting phase transformation upon charge and discharge. The multicomponent, multiphase-field model presented above features the advantage of decoupling interfacial and bulk energy contributions and, therefore, overcomes the discretization limitation of the classical Cahn-Hilliard description based on regular solution free energy. Computation of phase evolution within the Allen-Cahn framework enables the simulation of heterogeneous nucleation of a coherent LFP phase within a multigrain section of noncoherent FP phases. The model is able to predict composition-dependent phase transformation nucleating at grain boundaries without making a priori assumptions about the nucleation site. The multiphase field can be utilized to include lattice orientation information of the

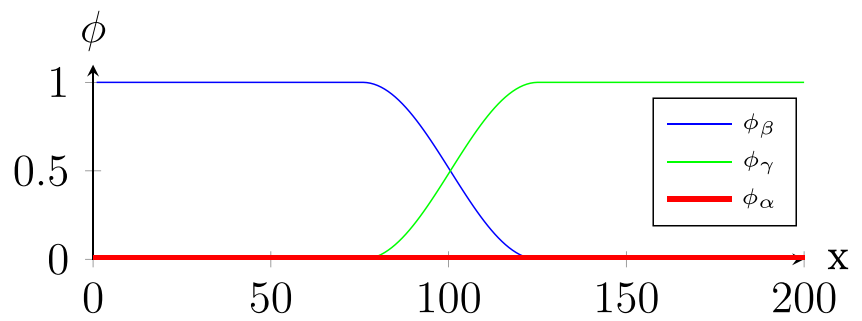

FIG. 12. $\phi_{\beta}-\phi_{\gamma}$ equilibrium interface. respective phases which can be reflected by anisotropy of diffusion and volumetric expansion. The explicit modeling of evolving phases also facilitates the incorporation of mechanical energy contributions based on mechanical jump conditions [40]. We intend to investigate these effects in future works.

The considerations in Sec. III have been utilized to relate the model parameters of the Cahn-Hilliard with the Allen-Cahn formulation but can further be applied for fitting parameters according to ab initio simulations or experimental results. Understanding the phase decomposition instability studied in Sec. IV A can be particularly useful for modeling solubility limits of the respective phases. We also note that this framework is not limited to a one-step phase transition process within the composition range of $\bar{c} \in[0,1]$ but can easily be extended to a multistep process as in the $\mathrm{FePO}_{4} \rightarrow$ $\mathrm{Na}_{0.66} \mathrm{FePO}_{4} \rightarrow \mathrm{NaFePO}_{4}$ phase transformation. Here we used a multiphase-field approach to study intercalation in a multigrain system. Simulations strongly support the assumption of coexistence of fully lithiated and delithiated primary particles as the filling process proceeds stepwise in a grainafter-grain manner through the agglomerate. This result is in agreement with the domino-cascade mechanism proposed by Delmas et al. [31] and the assumption that a core-shell-like intercalation occurs in larger secondary particles. Size and porosity of these secondary structures probably influences the battery performance strongly. The effects of lattice orientation, elastic energy, and the electrochemical reaction at active electrolyte-electrode surfaces on this process should be studied further.

\section{ACKNOWLEDGMENTS}

The authors thank P. Hoffrogge for the fruitful discussions. This work contributes to the research performed at CELEST (Center for Electrochemical Energy Storage Ulm-Karlsruhe) and was funded by the German Research Foundation Deutsche Forschungsgemeinschaft (DFG) under Project ID 390874152 (POLiS Cluster of Excellence). 


\section{APPENDIX A: STABILITY ANALYSIS IN A TWO-PHASE SYSTEM}

We introduce the system variable vector $y=[\phi, \bar{c}]^{T}$ and linearize the given system around the critical point $P_{0}=\left(\phi_{0}, c_{0}\right)$ following the approach in [57]:

$$
\dot{y}=\left[\begin{array}{cc}
-1 / \varepsilon \tau & 0 \\
0 & D \nabla^{2} / 2 A
\end{array}\right]\left(\left[\begin{array}{cc}
-2 \varepsilon \gamma_{\alpha \beta} & 0 \\
0 & 0
\end{array}\right]_{P_{0}} \nabla^{2} y+\left[\begin{array}{cc}
\frac{\partial^{2} f_{\text {pot }}}{\partial \phi^{2}}+\frac{\partial^{2} f_{\text {chem }}}{\partial \phi^{2}} & \frac{\partial^{2} f_{\text {chem }}}{\partial \phi \partial \bar{c}} \\
\frac{\partial^{2} f_{\text {chem }}}{\partial \phi \partial \bar{c}} & \frac{\partial^{2} f_{\text {chem }}}{\partial \bar{c}^{2}}
\end{array}\right]_{P_{0}} y\right) .
$$

We then transform our system into Fourier space with $\frac{\partial^{n} y}{\partial x^{n}}=(i k)^{n} \hat{y}(k)$,

$$
\frac{\partial \hat{y}}{\partial t}=-\left[\begin{array}{cc}
1 / \varepsilon \tau & 0 \\
0 & D k^{2} / 2 A
\end{array}\right]\left[\begin{array}{cc}
2 \varepsilon \gamma_{\alpha \beta} k^{2}+\frac{\partial^{2} f_{\text {pot }}}{\partial \phi^{2}}+\frac{\partial^{2} f_{\text {chem }}}{\partial \phi^{2}} & \frac{\partial^{2} f_{\text {chem }}}{\partial \phi \partial \bar{c}} \\
\frac{\partial^{2} f_{\text {chem }}}{\partial \phi \partial \bar{c}} & \frac{\partial^{2} f_{\text {chem }}}{\partial \bar{c}^{2}}
\end{array}\right]_{P_{0}} \hat{y} .
$$

As the gradient in $\phi$ is negligibly small initially, the gradient energy contribution can be neglected $|\nabla \phi|^{2} \approx 0$, which yields

$$
\frac{\partial \hat{y}}{\partial t}=-\tilde{M}\left[\begin{array}{cc}
\frac{\partial^{2} f_{\text {pot }}}{\partial \phi^{2}}+\frac{\partial^{2} f_{\text {chem }}}{\partial \phi^{2}} & \frac{\partial^{2} f_{\text {chem }}}{\partial \phi \partial \bar{c}} \\
\frac{\partial^{2} f_{\text {chem }}}{\partial \phi \partial \bar{c}} & \frac{\partial^{2} f_{\text {chem }}}{\partial \bar{c}^{2}}
\end{array}\right]_{P_{0}} \hat{y}=-\tilde{M} H_{f} \hat{y}
$$

with the system matrix $A=-\tilde{M} H_{f}$. The mobility matrix $\tilde{M}$ is diagonal and has two constant positive entries. The system is stable if the system matrix is negative definite which is fulfilled if the Hessian matrix $H_{f}$ is positive definite. We investigate the stability concerning small perturbations around a constant value of $\bar{c}$ by first looking at the determinant

$$
\operatorname{det}\left(H_{f}\right)=\left[\frac{\partial^{2} f_{\text {pot }}}{\partial \phi^{2}}+\frac{\partial^{2} f_{\text {chem }}}{\partial \phi^{2}}\right]\left[\frac{\partial^{2} f_{\text {chem }}}{\partial \bar{c}^{2}}\right]-\left[\frac{\partial^{2} f_{\text {chem }}}{\partial \bar{c} \partial \phi}\right]^{2}=\lambda_{1} \lambda_{2} .
$$

The entries of the Hessian matrix are

$$
\begin{aligned}
\frac{\partial^{2} f_{\text {tot }}}{\partial \phi^{2}} & =\frac{\partial^{2} f_{\text {pot }}}{\partial \phi^{2}}+2 A\left(\Delta c_{\text {min }}\right)^{2}\left(\frac{\partial h}{\partial \phi}\right)^{2}-2 A\left[\bar{c}-\bar{c}_{\min }(\phi)\right] \Delta c_{\min } \frac{\partial^{2} h}{\partial \phi^{2}}+B \frac{\partial^{2} h}{\partial \phi^{2}}, \\
\frac{\partial^{2} f_{\text {chem }}}{\partial \bar{c}^{2}} & =2 A, \frac{\partial^{2} f_{\text {chem }}}{\partial \bar{c} \partial \phi}=-2 A \Delta c_{\min } \frac{\partial h(\phi)}{\partial \phi}
\end{aligned}
$$

which yields with $\frac{\partial^{2} f_{\text {pot }}}{\partial \phi^{2}}=\frac{18 \gamma_{\alpha \beta}}{\varepsilon}\left(6 \phi^{2}-6 \phi+1\right)$ in the double-well case

$$
\operatorname{det}\left(H_{f}\right)=2 A \frac{18 \gamma_{\alpha \beta}}{\varepsilon}\left(6 \phi^{2}-6 \phi+1\right)+2 A B \frac{\partial^{2} h_{\mathrm{well}}}{\partial \phi^{2}}-4 A^{2}\left[\bar{c}-\bar{c}_{\min }(\phi)\right] \Delta c_{\min } \frac{\partial^{2} h_{\mathrm{well}}}{\partial \phi^{2}} \stackrel{!}{>} 0 .
$$

Investigating the stability of the critical solutions from Eq. (42) then leads to

$$
\frac{18 \gamma_{\alpha \beta}}{\varepsilon}-12 A\left(\bar{c}-c_{\min }^{\beta}\right) \Delta c_{\min }+6 B>0
$$

for $\phi=0, \bar{c}=c_{0}$ and from Eq. (43) yields

$$
\frac{18 \gamma_{\alpha \beta}}{\varepsilon}\left(6 \phi^{2}-6 \phi+1\right)-\frac{18 \gamma_{\alpha \beta}}{\varepsilon}(1-2 \phi)^{2}>0
$$

with $\phi \in(0,1)$. Evaluating Eq. (A2), the first critical solution becomes unstable for mole fractions higher than $c_{\text {crit }}$. Similar analysis can be performed for $\phi_{0}=1$ and we get

$$
\begin{aligned}
& \bar{c}_{\text {crit }}(\phi=0)=c_{\text {min }}^{\beta}+\frac{B}{2 A \Delta c_{\text {min }}}+\frac{3 \gamma_{\alpha \beta}}{2 A \Delta c_{\text {min }} \varepsilon}, \\
& c_{\text {crit }}(\phi=1)=c_{\text {min }}^{\alpha}+\frac{B}{2 A \Delta c_{\text {min }}}-\frac{3 \gamma_{\alpha \beta}}{2 A \Delta c_{\text {min }} \varepsilon} .
\end{aligned}
$$

In the second case, Eq. (A3) yields $-\phi(1-\phi)>0$ which is false $\forall \phi \in(0,1)$. This means the second critical solution is always unstable. To evaluate the stability of Eq. (A2) for $\bar{c}_{0}<\bar{c}_{\text {crit }}$ we look at the trace of the system $\operatorname{matrix} \operatorname{tr}\left(H_{f}\right)=\lambda_{1}+\lambda_{2}$ which yields for $\phi_{0}=0$

$$
2 A+\frac{18 \gamma_{\alpha \beta}}{\varepsilon}-12 A\left(\bar{c}_{0}-\bar{c}_{\min }^{\beta}\right) \Delta c_{\min }+6 B>0 .
$$

Expression (A4) holds $\forall \bar{c}_{0}<\bar{c}_{\text {crit }}$ and therefore is a stable solution of our system (40). 
In the case of an obstacle potential with $\frac{\partial^{2} f_{\mathrm{pot}}}{\partial \phi^{2}}=-\frac{32 \gamma_{\alpha \beta}}{\varepsilon \pi^{2}}$ the determinant reads

$$
\operatorname{det}\left(H_{f}\right)=-2 A \frac{32 \gamma_{\alpha \beta}}{\varepsilon \pi^{2}}+2 A B \frac{\partial^{2} h_{\mathrm{ob}}}{\partial \phi^{2}}-4 A^{2}\left[\bar{c}-\bar{c}_{\min }(\phi)\right] \Delta c_{\min } \frac{\partial^{2} h_{\mathrm{ob}}}{\partial \phi^{2}} \stackrel{!}{>} 0 .
$$

Inserting the critical points from Eq. (47), we get

$$
\operatorname{det}\left(H_{f}\right)=-\frac{8 \gamma_{\alpha \beta}}{\varepsilon \pi^{2}} \frac{1}{\phi(1-\phi)}<0 \quad \forall \phi \in(0,1),
$$

which means all critical solutions from Eq. (47) are unstable with respect to small fluctuations.

\section{APPENDIX B: STABILITY ANALYSIS OF THIRD-PHASE NUCLEATION WITHIN A PRESENT EQUILIBRIUM INTERFACE}

We assess the stability of an $\phi_{\beta}-\phi_{\gamma}$ equilibrium interface with regard to the formation of a third phase $\phi_{\alpha}$ driven by chemical energies depending on the global initial composition $\bar{c}_{0}$. The initial simulation setup is shown in Fig. 12 . Arguing that the two phases $\beta$ and $\gamma$ already present are equal in terms of stoichiometric composition and chemical energies, we assume $A^{\beta}=A^{\gamma}$, $c_{\min }^{\beta}=c_{\min }^{\gamma}=c_{\min }^{\beta \gamma}$, and $B^{\beta}=B^{\gamma}$. We further reduce complexity by choice of $A^{\alpha}=A^{\beta \gamma}=A$ and $B^{\alpha}=B, B^{\beta \gamma}=0$. The chemical energy Eq. (13) can be rewritten as a function of the average composition $\bar{c}=\sum c^{\alpha} h^{\alpha}(\boldsymbol{\phi})$ by introduction of $\Delta c_{\min }=c_{\min }^{\alpha}-c_{\min }^{\beta}$ and assuming equal phase diffusion potentials in the interface $\mu_{i}=\partial f^{\alpha} / \partial c_{i}^{\alpha}=\cdots=f^{N} / \partial c_{i}^{N}$,

$$
f_{\text {chem }}(\boldsymbol{\phi}, \bar{c})=A\left[\bar{c}-c_{\min }^{\beta \gamma}-\Delta c_{\min } h^{\alpha}(\boldsymbol{\phi})\right]^{2}+B h^{\alpha}(\boldsymbol{\phi}) .
$$

Due to the assumption of coherent nucleation, $\phi_{\alpha}$ only grows at the expense of $\phi_{\beta}$. We formulate the evolution equations for $\phi_{\alpha}$ based on Eq. (16):

$$
\begin{aligned}
\dot{\phi_{\alpha}}= & -\frac{M^{\alpha \beta}}{\tilde{N} \varepsilon}\left[\varepsilon \gamma_{\alpha \beta}\left(\nabla^{2} \phi_{\beta}-\nabla^{2} \phi_{\alpha}\right)+\frac{16}{\varepsilon \pi^{2}} \gamma_{\alpha \beta}\left(\phi_{\beta}-\phi_{\alpha}\right)+\varepsilon\left(\gamma_{\alpha \gamma}-\gamma_{\beta \gamma}\right)\left(\nabla^{2} \phi_{\gamma}+\frac{16}{\varepsilon \pi^{2}} \phi_{\gamma}\right)\right. \\
& \left.-2 A \Delta c_{\min }\left[\bar{c}-c_{\min }^{\beta}-\Delta c_{\min } h^{\alpha}(\boldsymbol{\phi})\right]\left(\frac{\partial h^{\alpha}}{\partial \phi_{\alpha}}-\frac{\partial h^{\alpha}}{\partial \phi_{\beta}}\right)+B\left(\frac{\partial h^{\alpha}}{\partial \phi_{\alpha}}-\frac{\partial h^{\alpha}}{\partial \phi_{\beta}}\right)\right]
\end{aligned}
$$

We assume $\nabla \phi_{\alpha}$ to be negligibly small, $\gamma_{\alpha \beta}$ equal for all phase pairs, and an equilibrium interface between the phases $\phi_{\beta}$ and $\phi_{\gamma}$. From the equilibrium solution $\phi_{\beta}=\frac{1}{2}+\frac{1}{2} \sin \left(\frac{4 x}{\varepsilon \pi}\right)$ we derive

$$
\frac{\partial^{2} \phi_{\beta}}{\partial x^{2}}=-\frac{8}{\varepsilon^{2} \pi^{2}} \sin \left(\frac{4 x}{\varepsilon \pi}\right)=\frac{8}{\varepsilon^{2} \pi^{2}}-\frac{16}{\varepsilon^{2} \pi^{2}} \phi_{\beta} .
$$

Employing Eq. (B3) the stationary solutions of Eq. (B2) are characterized by

$$
-\frac{8 \gamma}{\varepsilon \pi^{2}}\left(2 \phi_{\alpha}-1\right)-\left(2 A \Delta c_{\min }\left[\bar{c}-c_{\min }^{\beta}-\Delta c_{\min } h^{\alpha}(\boldsymbol{\phi})\right]-B\right)\left(\frac{\partial h^{\alpha}}{\partial \phi_{\alpha}}-\frac{\partial h^{\alpha}}{\partial \phi_{\beta}}\right) \stackrel{!}{=} 0
$$

which yields the criterion for stationary compositions

$$
\bar{c}_{\text {stat }}=c_{\min }^{\beta}+\frac{B}{2 A \Delta c_{\min }}+\Delta c_{\min } h^{\alpha}(\boldsymbol{\phi})+\frac{4 \gamma}{A \varepsilon \pi^{2} \Delta c_{\min }} \frac{1-2 \phi_{\alpha}}{\partial h^{\alpha} / \partial \phi_{\alpha}-\partial h^{\alpha} / \partial \phi_{\beta}} .
$$

The system evolution equation can be linearized around stationary point $P_{0}$ with the system variable vector $y=\left[\phi_{\alpha}, \bar{c}\right]^{T}$. We then transform our system into Fourier space with $\frac{\partial^{n} y}{\partial x^{n}}=(i k)^{n} \hat{y}(k)$ and get

$$
\frac{\partial \hat{y}}{\partial t}=-\left[\begin{array}{cc}
\frac{M^{\alpha \beta}}{\varepsilon \tilde{N}} & 0 \\
0 & \frac{D k^{2}}{2 A}
\end{array}\right]\left[\begin{array}{cc}
\varepsilon \gamma k^{2}-\frac{16 \gamma}{\varepsilon \pi^{2}}+\frac{\partial}{\partial \phi_{\alpha}}\left(\frac{\partial f_{\text {chem }}}{\partial \phi_{\alpha}}-\frac{\partial f_{\text {chem }}}{\partial \phi_{\beta}}\right) & \frac{\partial}{\partial \bar{c}}\left(\frac{\partial f_{\text {chem }}}{\partial \phi_{\alpha}}-\frac{\partial f_{\text {chem }}}{\partial \phi_{\beta}}\right) \\
\frac{\partial^{2} f_{\text {chem }}}{\partial \bar{c} \partial \phi_{\alpha}} & \frac{\partial^{2} f_{\text {chem }}}{\partial \bar{c}^{2}}
\end{array}\right]_{P_{0}} \hat{y}
$$

Assuming the gradient $\nabla \phi_{\alpha}$ to be negligibly small initially, this reduces to

$$
\frac{\partial \hat{y}}{\partial t}=-\tilde{M}\left[\begin{array}{cc}
-\frac{16 \gamma}{\varepsilon \pi^{2}}+\frac{\partial}{\partial \phi_{\alpha}}\left(\frac{\partial f_{\text {chem }}}{\partial \phi_{\alpha}}-\frac{\partial f_{\text {chem }}}{\partial \phi_{\beta}}\right) & \frac{\partial}{\partial \bar{c}}\left(\frac{\partial f_{\text {chem }}}{\partial \phi_{\alpha}}-\frac{\partial f_{\text {chem }}}{\partial \phi_{\beta}}\right) \\
\frac{\partial^{2} f_{\text {chem }}}{\partial \bar{c} \partial \phi_{\alpha}} & \frac{\partial^{2} f_{\text {chem }}}{\partial \bar{c}^{2}}
\end{array}\right]_{P_{0}} \hat{y}=-\tilde{M} S_{f} \hat{y}
$$

with the system matrix $A=-\tilde{M} S_{f}$. The mobility matrix $\tilde{M}$ is diagonal and has two constant positive entries. The system is stable if the stability matrix $S_{f}$ is positive definite. The determinant is given by

$$
\operatorname{det}\left(S_{f}\right)=S_{\phi_{\alpha} \phi_{\alpha}} S_{\bar{c} \bar{c}}-S_{\bar{c} \phi_{\alpha}} S_{\phi_{\alpha} \bar{c}}=\lambda_{1} \lambda_{2}
$$


with the single contributions

$$
\begin{gathered}
S_{\phi_{\alpha} \phi_{\alpha}}=-\frac{16 \gamma}{\varepsilon \pi^{2}}-2 A \Delta c_{\min }\left[\bar{c}-c_{\min }^{\beta}-\Delta c_{\min } h^{\alpha}(\boldsymbol{\phi})\right]\left(\frac{\partial^{2} h^{\alpha}}{\partial \phi_{\alpha}^{2}}-\frac{\partial^{2} h^{\alpha}}{\partial \phi_{\alpha} \partial \phi_{\beta}}\right)+2 A\left(\Delta c_{\min }\right)^{2} \frac{\partial h^{\alpha}}{\partial \phi_{\alpha}}\left(\frac{\partial h^{\alpha}}{\partial \phi_{\alpha}}-\frac{\partial h^{\alpha}}{\partial \phi_{\beta}}\right), \\
S_{\phi_{\alpha} \bar{c}}=-2 A \Delta c_{\min }\left(\frac{\partial h^{\alpha}}{\partial \phi_{\alpha}}-\frac{\partial h^{\alpha}}{\partial \phi_{\beta}}\right), \quad S_{\bar{c} \phi_{\alpha}}=-2 A \Delta c_{\min } \frac{\partial h^{\alpha}}{\partial \phi_{\alpha}}, \quad S_{\bar{c} \bar{c}}=2 A
\end{gathered}
$$

which yields

$$
\operatorname{det}\left(S_{f}\right)=-\frac{32 A \gamma}{\varepsilon \pi^{2}}-4 A^{2} \Delta c_{\min }\left[\bar{c}-c_{\min }^{\beta}-\Delta c_{\min } h^{\alpha}(\boldsymbol{\phi})\right]\left(\frac{\partial h^{\alpha}}{\partial \phi_{\alpha}^{2}}-\frac{\partial h^{\alpha}}{\partial \phi_{\alpha} \phi_{\beta}}\right) \stackrel{!}{>} 0 .
$$

Investigating the stability of the critical solutions from Eq. (B4), we derive the criterion

$$
-\frac{16 \gamma}{\varepsilon \pi^{2}}-\frac{8 \gamma}{\varepsilon \pi^{2}} \frac{1-2 \phi_{\alpha}}{\partial h^{\alpha} / \partial \phi_{\alpha}-\partial h^{\alpha} / \partial \phi_{\beta}}\left(\frac{\partial h^{\alpha}}{\partial \phi_{\alpha}^{2}}-\frac{\partial h^{\alpha}}{\partial \phi_{\alpha} \phi_{\beta}}\right)>0 .
$$

[1] B. Han, A. Van Der Ven, D. Morgan, and G. Ceder, Electrochim. Acta 49, 4691 (2004).

[2] J. E. Guyer, W. J. Boettinger, J. A. Warren, and G. B. McFadden, Phys. Rev. E 69, 021603 (2004).

[3] L. Liang, Y. Qi, F. Xue, S. Bhattacharya, S. J. Harris, and L.-Q. Chen, Phys. Rev. E 86, 051609 (2012).

[4] D. A. Cogswell, Phys. Rev. E 92, 011301(R) (2015).

[5] Z. Hong and V. Viswanathan, ACS Energy Lett. 3, 1737 (2018).

[6] G. Singh, G. Ceder, and M. Bazant, Electrochim. Acta 53, 7599 (2008).

[7] P. Bai, D. Cogswell, and M. Bazant, Nano Lett. 11, 4890 (2011).

[8] D. Cogswell and M. Bazant, ACS Nano 6, 2215 (2012).

[9] D. Cogswell and M. Bazant, Electrochem. Commun. 95, 33 (2018).

[10] M. Tang, J. Belak, and M. Dorr, J. Phys. Chem. C 115, 4922 (2011).

[11] L. Hong, L. Li, Y. K. Chen-Wiegart, J. Wang, K. Xiang, L. Gan, W. Li, F. Meng, F. Wang, J. Wang, Y. Chiang, S. Jin, and M. Tang, Nat. Commun. 8, 1194 (2017).

[12] L. Hong, K. Yang, and M. Tang, npj Comput. Mater. 5, 1 (2019).

[13] M. Huttin and M. Kamlah, Appl. Phys. Lett. 101, 133902 (2012).

[14] A. Walk, M. Huttin, and M. Kamlah, Eur. J. Mech., A-Solids 48, 74 (2014).

[15] T. Zhang and M. Kamlah, J. Electrochem. Soc. 165, A1997 (2018).

[16] T. Zhang and M. Kamlah, Electrochim. Acta 298, 31 (2019).

[17] Y. Zhao, P. Stein, and B.-X. Xu, Comput. Methods Appl. Mech. Eng. 297, 325 (2015).

[18] Y. Zhao, B.-X. Xu, P. Stein, and D. Gross, Comput. Methods Appl. Mech. Eng. 312, 428 (2016).

[19] J. Santoki, M. Kamlah, D. Schneider, B. Nestler, M. Selzer, and F. Wang, Modell. Simul. Mater. Sci. Eng. 26, 065013 (2018).

[20] L. Hong, L. Liang, S. Bhattacharyya, W. Xing, and L. Chen, Phys. Chem. Chem. Phys. 18, 9537 (2016).

[21] Y. Zhao, Phase-field modeling of electro-chemo-mechanical behavior of li-ion battery electrodes, Ph.D. thesis, Technische Universität Darmstadt, 2017.
[22] Y. Zhao, L. De Jesus, P. Stein, G. Horrocks, S. Banerjee, and B.-X. Xu, RSC Adv. 7, 41254 (2017).

[23] L. Wu, V. De Andrade, X. Xiao, and J. Zhang, J. Electrochemical Energy Convers. Storage 16, 041006 (2019).

[24] A. Padhi, K. Nanjundaswamy, and J. Goodenough, J. Electrochem. Soc. 144, 1188 (1997).

[25] A. Yamada, H. Koizumi, N. Sonoyama, and R. Kanno, Electrochem. Solid State Lett. 8 (2005).

[26] G. Chen, X. Song, and T. Richardson, Electrochem. Solid State Lett. 9, 295 (2006).

[27] A. Abdellahi, O. Akyildiz, R. Malik, K. Thornton, and G. Ceder, J. Mater. Chem. A 2, 15437 (2014).

[28] N. G. Hörmann and A. Groß, Phys. Rev. Materials 3, 055401 (2019).

[29] V. Srinivasan and J. Newman, J. Electrochem. Soc. 151, A1517 (2004).

[30] M. Bazant, Acc. Chem. Res. 46, 1144 (2013).

[31] C. Delmas, M. Maccario, L. Croguennec, F. Le Cras, and F. Weill, Nat. Mater. 7, 665 (2008).

[32] S. Bhattacharyya, S. Bandyopadhyay, and A. Choudhury, J. Indian Inst. Sci. 96, 257 (2016)

[33] M. Fleck, H. Federmann, and E. Pogorelov, Comput. Mater. Sci. 153, 288 (2018).

[34] I. Steinbach, F. Pezzolla, B. Nestler, M. Seeßelberg, R. Prieler, G. Schmitz, and J. Rezende, Physica D 94, 135 (1996).

[35] B. Nestler, H. Garcke, and B. Stinner, Phys. Rev. E 71, 041609 (2005).

[36] M. Plapp, Phys. Rev. E 84, 031601 (2011).

[37] A. Choudhury and B. Nestler, Phys. Rev. E 85, 021602 (2012).

[38] R. Folch and M. Plapp, Phys. Rev. E 72, 011602 (2005).

[39] D. Schneider, O. Tschukin, A. Choudhury, M. Selzer, T. Böhlke, and B. Nestler, Comput. Mech. 55, 887 (2015).

[40] D. Schneider, E. Schoof, O. Tschukin, A. Reiter, C. Herrmann, F. Schwab, M. Selzer, and B. Nestler, Comput. Mech. 61, 277 (2018).

[41] P. Kubendran Amos, E. Schoof, D. Schneider, and B. Nestler, J. Alloys Compd. 767, 1141 (2018). 
[42] L. K. Aagesen, Y. Gao, D. Schwen, and K. Ahmed, Phys. Rev. E 98, 023309 (2018).

[43] J. Cahn and J. Hilliard, J. Chem. Phys. 28, 258 (1958).

[44] J. Hötzer, A. Reiter, H. Hierl, P. Steinmetz, M. Selzer, and B. Nestler, J. Comput. Sci. 26, 1 (2018).

[45] P. Boyanova and M. Neytcheva, Comput. Math. Appl. 67, 106 (2014).

[46] I. Steinbach and F. Pezzolla, Physica D 134, 385 (1999).

[47] G. I. Tóth, T. Pusztai, and L. Gránásy, Phys. Rev. B 92, 184105 (2015).

[48] I. Steinbach, Modell. Simul. Mater. Sci. Eng. 17, 073001 (2009).

[49] N. Moelans, Acta Mater. 59, 1077 (2011).
[50] E. Schoof, D. Schneider, N. Streichhan, T. Mittnacht, M. Selzer, and B. Nestler, Int. J. Solids Struct. 134, 181 (2018).

[51] M. Kellner, J. Hötzer, E. Schoof, and B. Nestler, Acta Mater. 182, 267 (2020).

[52] J. Cahn and J. Hilliard, J. Chem. Phys. 31, 688 (1959).

[53] J. Cahn, Acta Metall. 9, 795 (1961).

[54] J. Cahn, J. Chem. Phys. 42, 93 (1965).

[55] S. G. Kim, W. T. Kim, and T. Suzuki, Phys. Rev. E 60, 7186 (1999).

[56] See Supplemental Material at http://link.aps.org/supplemental/ 10.1103/PhysRevMaterials.5.035406 for temporal evolution of concentration and phases during the intercalation process.

[57] M. San Miguel, J. D. Gunton, G. Dee, and P. S. Sahni, Phys. Rev. B 23, 2334 (1981). 